\title{
Total correlation spectroscopy across all NMR-active nuclei by mixing at zero field
}

\author{
Ivan V. Zhukova ${ }^{a, b}$, Alexey S. Kiryutinn ${ }^{a, b}$, Fabien Ferrage ${ }^{c}$, Gerd Buntkowsky ${ }^{d}$, \\ Alexandra V. Yurkovskayaa, ${ }^{a, b}$ Konstantin L. Ivanov ${ }^{a, b, *}$
}

\author{
a International Tomography Center SB RAS, Novosibirsk 630090, Russia \\ ${ }^{b}$ Novosibirsk State University, Novosibirsk 630090, Russia \\ ' Laboratoire des Biomolécules, Département de chimie, École normale supérieure, PSL University, \\ Sorbonne Université, CNRS, 75005 Paris, France \\ d Technical University of Darmstadt, Institute of Inorganic and Physical Chemistry, D-64287 \\ Darmstadt, Germany ORCID: 0000-0003-1304-9762
}

* Corresponding author, email: ivanov@tomo.nsc.ru

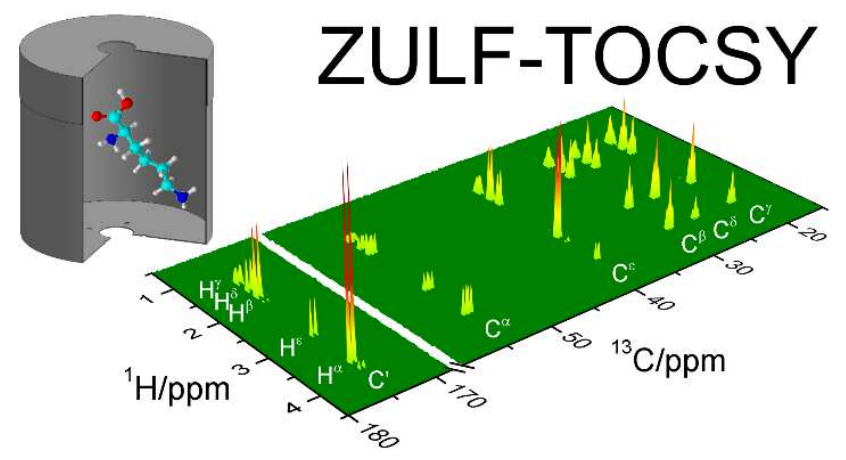

Abstract

Multidimensional Nuclear Magnetic Resonance (NMR) is based on the combination of well-established building blocks for polarization transfer. These blocks are used to design correlation experiments through one or a few chemical bonds or through space. Here, we introduce a building block that enables polarization transfer across all NMR-active nuclei in a coupled network of spins: isotropic mixing at Zero and Ultra-Low Field (ZULF). Exploiting mixing under ZULF-NMR conditions, heteronuclear TOtal Correlation SpectroscopY (TOCSY) experiments were developed to highlight coupled spin networks. We demonstrate ${ }^{1} \mathrm{H}-{ }^{13} \mathrm{C}$ and ${ }^{1} \mathrm{H}_{-}{ }^{15} \mathrm{~N}$ correlations in ZULF-TOCSY spectra of labelled amino acids, which allow one to obtain cross-peaks among all hetero-nuclei belonging to the same coupled network, even when the direct interaction between them is negligible. We also demonstrate the interest of ZULF-TOCSY to analyze complex mixtures on the supernatant of ISOGRO, a growth medium of isotope-labelled biomolecules. ZULF-TOCSY enables the quick identification of individual compounds in the mixture by their coupled spin networks. The ZULF-TOCSY method will lead to the development of a new toolbox of experiments to analyze complex mixtures by NMR. 


\section{Introduction}

Two dimensional Nuclear Magnetic Resonance (2D-NMR) ${ }^{1}$ has revolutionized NMR spectroscopy by introducing powerful methods to greatly increase spectral resolution, provide topological information to easily assign signals of complex molecules and structural constraints to refine the conformations of small and macro-molecules. As such, 2D NMR has had a deep impact in synthetic and materials chemistry, as well as structural biology. All 2D-NMR experiments are performed with the following steps (Figure 1a): preparation of the nuclear spin coherence of interest (step 1), evolution of this coherence under an interaction during the time $t_{1}$ (step 2), mixing (step 3), observation of the Free Induction Decay (FID) signal as a function of time $t_{2}$ (step 4). Repeating the experiment multiple times with variable time $t_{1}$ provides the signal intensity in two time dimensions, $S\left(t_{1}, t_{2}\right)$, which gives a spectrum in two dimensions after Fourier transformation.

The specific choice of the mixing block is central as it defines the nature of the correlation between the two frequency dimensions, i.e. the type of information that the 2D-NMR will provide. For instance, crosspeaks in $\mathrm{COSY},{ }^{2} \mathrm{HSQC}^{3} \mathrm{HMQC}^{4}$ and $\mathrm{HMBC}^{5}$ experiments originate from scalar spin-spin interactions among homonuclei and heteronuclei, and so indicate topological connections through covalent bonds or hydrogen bonds, ${ }^{6-8}$ between atoms. On the other hand, nuclear Overhauser spectroscopy (NOESY) experiments reports on cross-relaxation between nuclear spins through space, which can be converted to distances between atoms. ${ }^{9}$ One of the most widely used 2D-NMR methods is TOtal Correlation SpectroscopY (TOCSY), ${ }^{10}$ which is designed to elucidate scalar-coupled spin networks. In the TOCSY case, the mixing is obtained by using a strong radiofrequency (RF) pulse, which gives rise to isotropic mixing among homonuclei. As a consequence, all spins belonging to the same coupling network exhibit crosspeaks. The TOCSY method is very useful for assigning signals in molecules constituted of assembled units, such as proteins, ${ }^{11}$ nucleic acids, ${ }^{12}$ and polysaccharides. ${ }^{13}$ Similarly, the TOCSY experiment is extremely valuable for the investigation of complex mixtures of small molecules. ${ }^{14}$

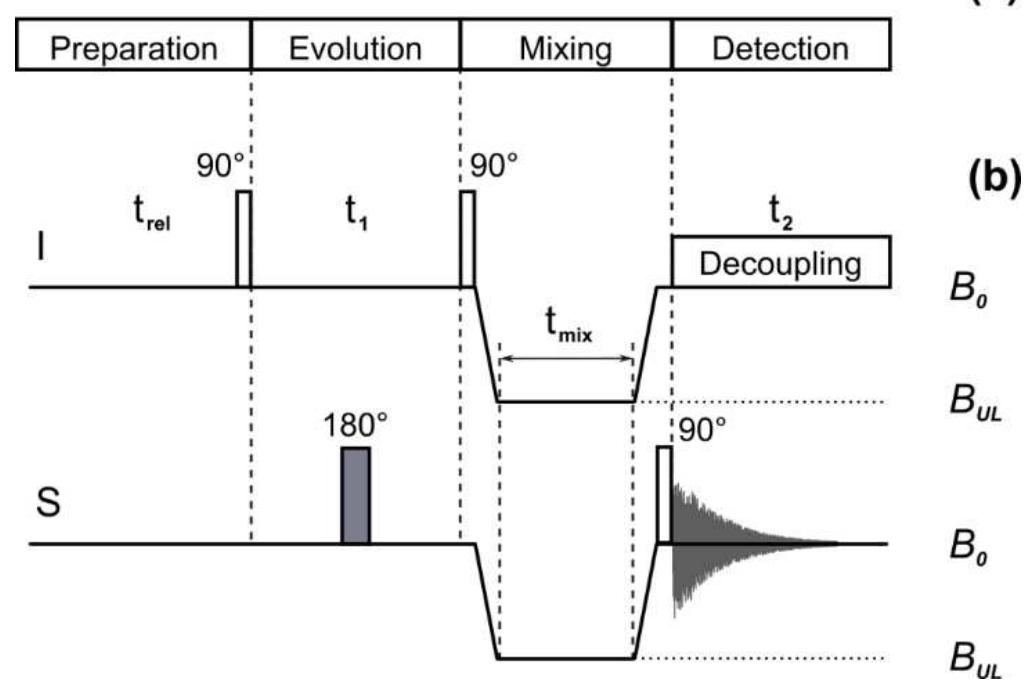

Figure 1. (a) Stages of a general 2D NMR experiment: preparation of an initial spin order; evolution during a variable time period $t_{1}$; mixing block converting spin order into observable NMR signal; detection of a time domain signal as a function of $t_{2}$ time. (b) Protocol of the heteronuclear ZULF-TOCSY experiment. A 90-degree pulse after a suitable relaxation delay $t_{\text {rel }}$ generates transverse order of the $I$ spins (here protons). This spin order evolves under chemical shift Hamiltonian during the period $t_{1}$, mapping the indirect spectrum dimension. Heteronuclear J-coupling is refocused by a 180-degree pulse on the $S$ spin channel (here $\mathrm{S}$ spins ${ }^{13} \mathrm{C}$ and ${ }^{15} \mathrm{~N}$ have been used) placed at $t=t_{1} / 2$. At $t=t_{1}$ the transverse polarization of the $I$ spins is converted back into longitudinal polarization. The mixing block consists of a field switch $B_{0} \leftrightarrow B_{U L}$, lasting for $t_{s w} \approx 0.4 \mathrm{~s}$, and isotropic mixing under ZULF conditions for $t_{\text {mix }}$ of several $10 \mathrm{~ms}$. When the FID signal of $S$ spins is acquired, i.e., the time domain signal in the direct dimension, composite pulse decoupling on the $I$-channel is applied.

The HMBC experiment gives information about correlations of unlike spins through a few bonds (usually 2 to 3), the heteronuclear cross-polarization, also called heteronuclear Hartmann-Hahn (HEHAHA) 
provides correlations between two types of nuclei, with limitations on bandwidth ${ }^{15-17}$ and threedimensional experiments such as TOCSY-HSQC ${ }^{18}$ and TOCSY-HMQC ${ }^{19}$ combine some features of TOCSY with correlations between proton-bound heteronuclei. Yet, there is, to date, no experiment that provides directly full correlations across network of coupled like and unlike nuclei. Here, we introduce a new concept in total correlation spectroscopy, providing correlations between all NMR-active nuclei in a coupled network. We achieve isotropic mixing across all nuclei by transferring the sample to Zero and Ultra-Low Field (ZULF) NMR ${ }^{20}$ conditions using a sample shuttle, ${ }^{21-24}$ while all chemical-shift evolutions are carried out at high field on a high-resolution NMR spectrometer (Figure 1b). Zero or ultra-low field TOCSY (ZULF-TOCSY) enables isotropic mixing among heteronuclei combined with sensitivity and excellent spectral resolution provided by modern high-field NMR methods. We illustrate the method on isotopically enriched amino acids, and demonstrate that ZULF-TOCSY allows the efficient assignment of signals of individual molecules in complex mixtures.

\section{Methods}

Experiments were carried out on aqueous solutions of uniformly ${ }^{13} \mathrm{C}$ and ${ }^{15} \mathrm{~N}$ labelled amino acids, Lleucine (Sigma-Aldrich, product no. 608068; $100 \mathrm{mM}, \mathrm{pH}$ 5.9) and L-lysine (Sigma-Aldrich, product no. $608041 ; 100 \mathrm{mM}, \mathrm{pH}$ 5.7), as well as ISOGRO (MERCK; pH = 6.5) growth medium solubilized in deuterated water (Deutero $\mathrm{GmbH}$ ). ISOGRO is a complex mixture of uniformly isotopically enriched ${ }^{13} \mathrm{C}$ and ${ }^{15} \mathrm{~N}$ molecules: amino acids and small peptides (65\%), glucose (2\%), as well as salts (30\%). ${ }^{25} \mathrm{The} \mathrm{pH}$ of aqueous solution was measured by electrode calibrated in protonated water. All experiments were carried out at $300 \mathrm{~K}$.

NMR experiments were performed using a commercial 9.4 T NMR spectrometer (Avance III HD, Bruker) equipped by TXI and BBO probes. The spectrometer was augmented with an add-on device to shuttle the sample along the vertical axis between the detection point at 9.4 $\mathrm{T}$ and a magnetic shield with a magnetic field strength inside in the range $10 \mathrm{nT}-25 \mu \mathrm{T} .{ }^{26} \mathrm{To}$ implement non-adiabatic field variation we used the same method as described before ${ }^{26}$ with mechanical sample transport to an intermediate guiding field $B_{\text {int }} \approx 25 \mu \mathrm{T}$ within $0.4 \mathrm{~s}$, which is rapidly (within less than $100 \mu \mathrm{s}$ ) reduced to a $B_{U L}$ field of desired strength; the $B_{U L} \rightarrow B_{0}$ switch is done in the same way, $B_{U L} \rightarrow B_{\text {int }} \rightarrow B_{0}$.

Chemical shift referencing for proton spectra was done by referencing of residual water signal. The absolute water chemical shift was calculated using the formula $\delta(\mathrm{HDO})=7.83-T / 96.9,{ }^{27}$ where $T$ is the absolute temperature; in the present case $\delta(\mathrm{HDO})$ was $4.73 \mathrm{ppm}$. Referencing of the ${ }^{13} \mathrm{C}$ and ${ }^{15} \mathrm{~N}$ resonance frequencies was done indirectly according to recommendations for biomolecular $\mathrm{NMR}^{28}$ in the Mnova software (version 12).

\section{Results and Discussion}

The ZULF-TOCSY experiment was designed to achieve efficient isotropic mixing between heteronuclei and high-resolution chemical shift evolution in all dimensions. Here, we describe the ZULF-TOCSY pulse sequence in the case of a heteronuclear spin system comprising $I$ spins and $S$ spins (Figure 1b). High sensitivity is obtained by polarizing the sample at a high magnetic field $B_{0}$. Chemical shift evolution for the $I$ spins is obtained during the time $t_{1}$, during which an inversion pulse on the $S$ spins refocuses the evolution under heteronuclear couplings. At $t=t_{1}$ transverse polarization is converted back to longitudinal polarization before a fast (non-adiabatic) field jump from the high-field $B_{0}$ to the ultralow field $B_{U L}$ of about $100 \mathrm{nT}$ for mixing (as explained above). Polarization transfer throughout all coupling networks is allowed at the ultra-low field during a time $t_{m i x}$. The sample is transferred back to the $B_{0}$ field by a fast non-adiabatic field jump $B_{U L} \rightarrow B_{0}$ to detect at high field for optimal resolution on the $S$-channel. We would like to stress that despite the apparent similarity of the pulse sequence to heteronuclear NOESY, spin mixing works in a very different way and the mechanism of polarization transfer between heteronuclei is different (coherent polarization transfer rather than cross-relaxation). 
The ZULF-TOCSY method relies on efficient isotropic mixing at the ultralow magnetic field $B_{U L}$. Here, we briefly explain the coherent mechanism for isotropic mixing. The Hamiltonian of the spin system at $B=$ $B_{U L}$ is written as follows (in $\hbar$ units):

$$
\widehat{\mathcal{H}}=\omega_{I} \sum_{i} \hat{I}_{i z}+\omega_{S} \sum_{k} \hat{S}_{k z}+2 \pi \sum_{i>j} J_{i j}^{I I}\left(\hat{\mathbf{I}}_{i} \cdot \hat{\mathbf{I}}_{j}\right)+2 \pi \sum_{k>l} J_{i j}^{S S}\left(\hat{\mathbf{S}}_{k} \cdot \hat{\mathbf{S}}_{l}\right)+2 \pi \sum_{i, k} J_{i j}^{I S}\left(\hat{\mathbf{I}}_{i} \cdot \hat{\mathbf{S}}_{k}\right)
$$

Here $\omega_{I, S}=-\gamma_{I, S} \times B_{U L}$ is the angular frequency of the Zeeman interaction of spins $I, S$ with the gyromagnetic ratio $\gamma_{I, S}$. Here we neglect the chemical shifts of individual nuclei. $J_{i j}^{I I}, J_{k l}^{S S}, J_{i k}^{I S}$ are the homonuclear and heteronuclear scalar-coupling constants. It the situation where the difference in Larmor frequency $\left\{\omega_{I}-\omega_{S}\right\} / 2 \pi$ becomes much smaller than the heteronuclear coupling constants, $J_{i k}^{I S}$, there is no longer a difference between homo- and heteronuclear spin. ${ }^{29-34}$ The strong coupling conditions are identical to those in homonuclear TOCSY experiments, ${ }^{10}$ in which the chemical shift differences are quenched for homonuclei by applying a strong radiofrequency field. Thus, at $B=B_{U L}$ polarization transfer via an entire coupling network can be achieved. For $B_{U L}=100 \mathrm{nT}$ the Larmor frequencies of ${ }^{1} \mathrm{H}$, ${ }^{13} \mathrm{C}$ and ${ }^{15} \mathrm{~N}$ are $4.26 \mathrm{~Hz}, 1.07 \mathrm{~Hz}$ and $0.43 \mathrm{~Hz}$, respectively, whereas typical values of the direct scalar coupling constants are ${ }^{1} J\left({ }^{13} \mathrm{C},{ }^{1} \mathrm{H}\right) \sim 140 \mathrm{~Hz}$ for a quaternary carbon and ${ }^{1} J\left({ }^{15} \mathrm{~N},{ }^{1} \mathrm{H}\right) \sim 7 \mathrm{~Hz}$ in the amino group. The homonuclear ${ }^{1} \mathrm{H}$ and ${ }^{13} \mathrm{C}$ subsystems are expected to be in the strong coupling regime during the field switching step at significantly higher fields, being in the $\mathrm{mT}$ range. Hence, at $B_{U L}=100$ $\mathrm{nT}$ isotropic mixing of the entire spin network is expected.

To implement the ZULF-TOCSY method we used a $400 \mathrm{MHz}$ NMR spectrometer equipped with a fast fieldcycling device, ${ }^{26}$ which allows one to perform magnetic field switches $B_{0} \leftrightarrow B_{U L}$ in approximately $0.4 \mathrm{~s}$; which is non-adiabatic for the heteronuclei. ${ }^{35}$ Performing fast field jumps is a prerequisite for ZULF-TOCSY. Non-adiabatic field jumps turn the difference in the polarizations of the $I$ spins and $S$ spins into heteronuclear zero-quantum coherences (ZQCs), which mediate the polarization transfer; the ZQC evolution is driven by scalar spin-spin interactions. ${ }^{26,36}$

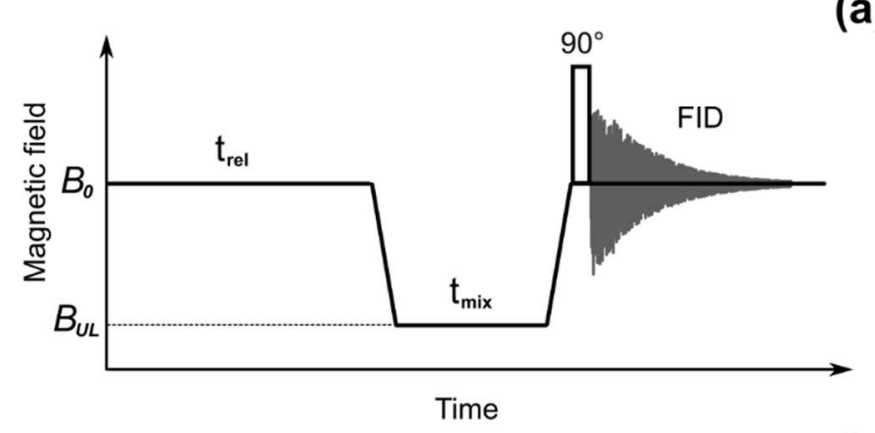

(a)

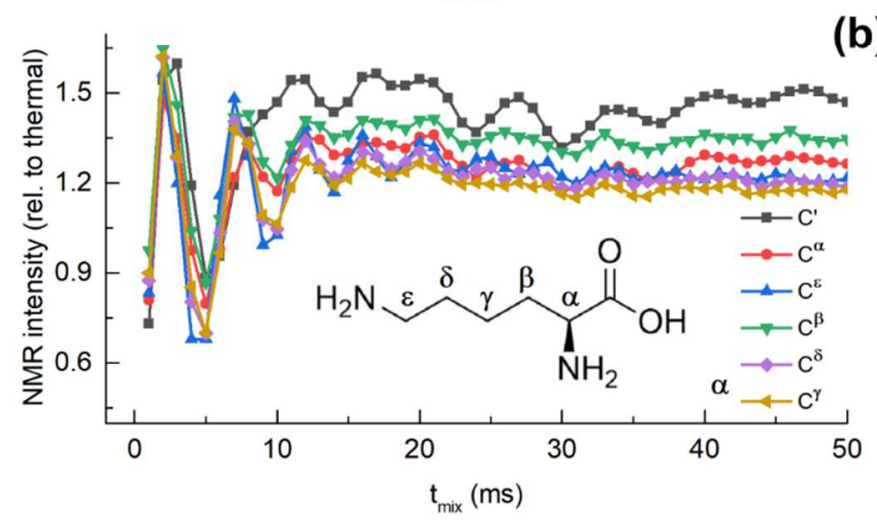

Figure 2. (a) Protocol of the ZULF polarization transfer experiment. Spin system with equilibrium magnetization is transferred non-adiabatically to an ultralow field $B_{U L}$. After evolution caused by difference in population of spins I and $\mathrm{S}$ during $t_{\text {mix }}$ field is switched back to $B_{0}$ where FID of either spin I or spin $\mathrm{S}$ is detected. Varying $t_{m i x}$ one can obtain the kinetics of the polarization transfer between proton (I spins) and heteronuclei ( $S$ spin). (b) Dependence of ${ }^{13} \mathrm{C}$ NMR signal intensities of uniformly ${ }^{13} \mathrm{C},{ }^{15} \mathrm{~N}$ labeled L-lysine on $t_{\text {mix }}$ obtained for $B_{U L}=100 \mathrm{nT}$. The resulting signals are normalized to the thermal signals at $B=B_{0}$. 
We have optimized the ZULF-TOCSY mixing time with a simple field-jump experiment (Figure 2a). Recovery was allowed for all nuclei (e.g. protons, ${ }^{13} \mathrm{C}$, and ${ }^{15} \mathrm{~N}$ ) at high magnetic field, $B=B_{0}$ to reach their equilibrium polarizations, which is different for different spin isotopes due to the difference in their gyromagnetic ratios. Subsequently, a non-adiabatic field jump to the ultralow field $B_{U L}$ converts the difference in spin polarizations into ZQCs, which evolve during a variable time $t_{\text {mix }}$. The amount of polarization transfer was measured after a non-adiabatic field jump back to high field $B_{U L} \rightarrow B_{0}$, which converts ZQCs back into longitudinal polarizations, right before detection. Polarization transfer from ${ }^{1} \mathrm{H}$ to ${ }^{13} \mathrm{C}$ nuclei in uniformly ${ }^{13} \mathrm{C},{ }^{15} \mathrm{~N}$-labeled L-lysine is occurring on the timescale of around $10 \mathrm{~ms}$ (Figure $2 \mathrm{~b}$ ). The polarization transfer from protons leads to stronger signals of ${ }^{13} \mathrm{C}$ than those obtained at thermal equilibrium at $B=B_{0}$. The $t_{m i x}$ dependence of polarization contains fast oscillations, which are due to the evolution of the ZQCs, driven by J-couplings, confirming efficient coherent polarization transfer among hetero-nuclei at $B=B_{U L}$. Similar results were obtained for ${ }^{1} \mathrm{H}$ to ${ }^{13} \mathrm{C}$ polarization transfer in L-leucine, as well as for polarization transfer among protons and ${ }^{15} \mathrm{~N}$ nuclei in Lysine (see Supporting Information). The $t_{m i x}$ value of $2 \mathrm{~ms}$, corresponding to the first maximum in the oscillatory curves shown in Figure $2 \mathrm{~b}$, is usually sufficient for achieving polarization transfer at the $B_{U L}$ field, i.e., for obtaining ZULF-TOCSY crosspeaks (experiments with longer $t_{\text {mix }}$ times give similar results). In the case of ${ }^{1} \mathrm{H}-{ }^{15} \mathrm{~N}$ correlation longer $t_{m i x}$, typically about $50 \mathrm{~ms}$, are required.

(a)
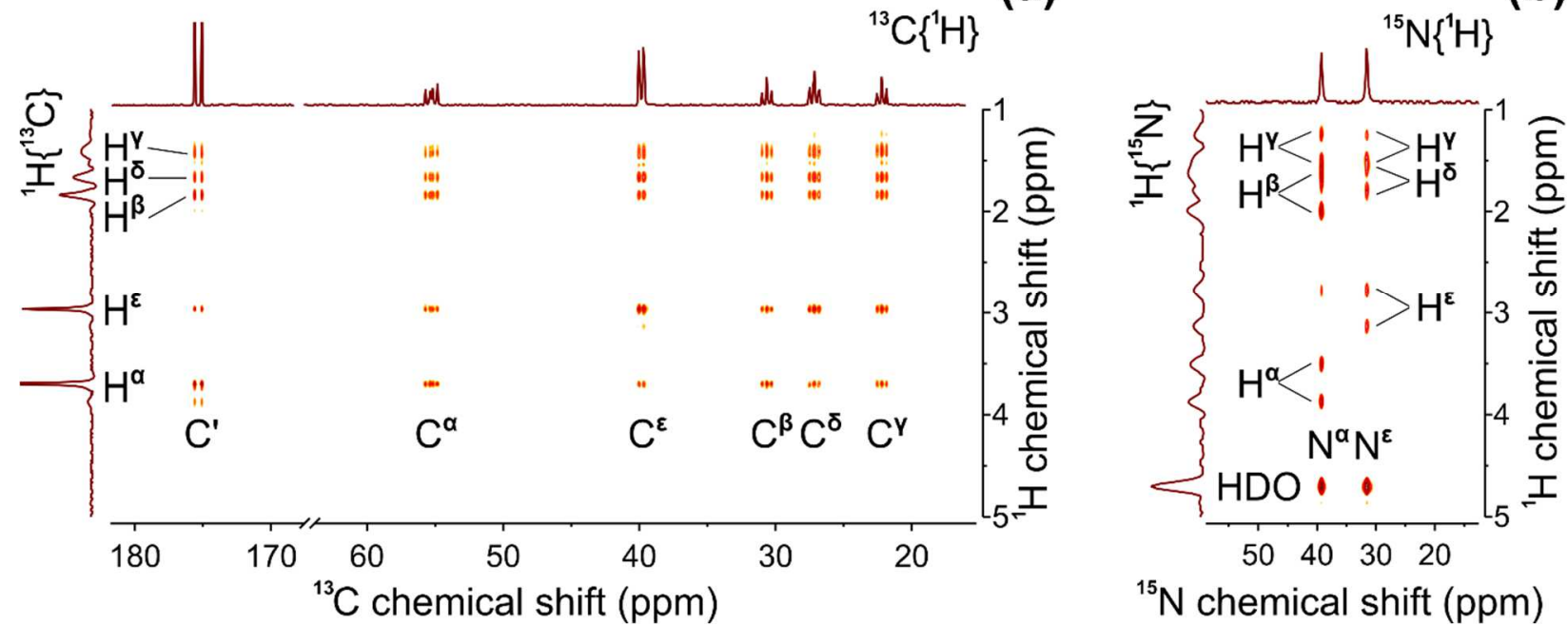

Figure 3. (a) ${ }^{13} \mathrm{C}-1 \mathrm{H}$ ZULF-TOCSY spectrum of ${ }^{13} \mathrm{C},{ }^{15} \mathrm{~N}$-labeled L-lysine. Experimental parameters: $B_{U L}=100 \mathrm{nT}, t_{\text {mix }}=2 \mathrm{~ms}, 128$ transients in indirect direction, 4 scans per transient, relaxation delay $6 \mathrm{~s}$, total experiment duration ca. 70 minutes. Linear prediction of the indirect dimension FID points 129-512 was applied using the Zhu-Bax ${ }^{37}$ method. Acquired data multiplied by $90^{\circ}$ shifted squared sinusoidal window function in both dimensions. Spectrum presented by $2048 \times 512$ data points. (b) ${ }^{15} \mathrm{~N}-1 \mathrm{H}$ ZULF-TOCSY spectrum of ${ }^{13} \mathrm{C},{ }^{15} \mathrm{~N}$ labeled L-lysine. Experimental parameters: $B_{U L}=100 \mathrm{nT}, t_{\text {mix }}=50 \mathrm{~ms}, 64$ transients in indirect direction, 128 scans per transient, relaxation delay $23 \mathrm{~s}$, total experiment duration ca. 62 hours. Linear prediction of indirect dimension FID points 64-256 was applied using the Zhu-Bax ${ }^{37}$ method. Acquired data multiplied by a $90^{\circ}$-shifted squared sinusoidal window function in both dimensions. The spectrum is given by $2048 \times 256$ data points. Splitting in the indirect dimension is due to a non-refocused scalar coupling with the ${ }^{13} \mathrm{C}$ nuclei, to which the proton is attached.

The ZULF-TOCSY spectra of L-lysine display extensive ${ }^{1} \mathrm{H}-{ }^{13} \mathrm{C}$ and ${ }^{1} \mathrm{H}-{ }^{15} \mathrm{~N}$ correlations (Figure 3). Polarization is transferred through the entire spin system, under isotropic mixing conditions at the ultralow field $B=$ $B_{U L}$. In particular, the ${ }^{1} \mathrm{H}^{13} \mathrm{C}$ spectrum shows that all proton peaks are correlated to all carbon peaks, since all of them belong to the same coupling network. For instance, the $\varepsilon-\mathrm{CH}_{2}$ protons exhibit cross-peaks with all carbons, although sizeable direct coupling is expected only for the carbon nuclei in the $\varepsilon$ and $\delta$ positions. At the same time, only the $\mathrm{H} \varepsilon$-C $\varepsilon$ correlation can be observed in the HSQC spectrum (see Supporting Information). It is also important to point out that the $C^{\prime}$ carbon, which does not exhibit any cross-peak in the HSQC spectrum (because of the absence of one-bond coupling to a proton), displays cross-peaks with all protons in the ZULF-TOCSY spectrum. In the ${ }^{1} \mathrm{H}-{ }^{15} \mathrm{~N}$ ZULF-TOCSY spectra one can also see cross-peaks of both ${ }^{15} \mathrm{~N}$ nuclei with most protons, including remote protons separated from the ${ }^{15} \mathrm{~N}$ 
atoms by four bonds, which results in negligible direct couplings. By contrast, only two- and three-bond correlations can be observed in the HSQC spectrum with exceptionally long INEPT transfer times (Supporting Information). Interestingly, correlations with the signal of HOD demonstrates combined polarization transfer by isotropic mixing and chemical exchange of labile protons. The ZULF-TOCSY results for the uniformly labelled L-leucine, are qualitatively similar (Supporting Information). Detection of ZULFTOCSY spectra on the X-channel provides excellent resolution in the direct ${ }^{13} \mathrm{C}$ or ${ }^{15} \mathrm{~N}$ dimension.

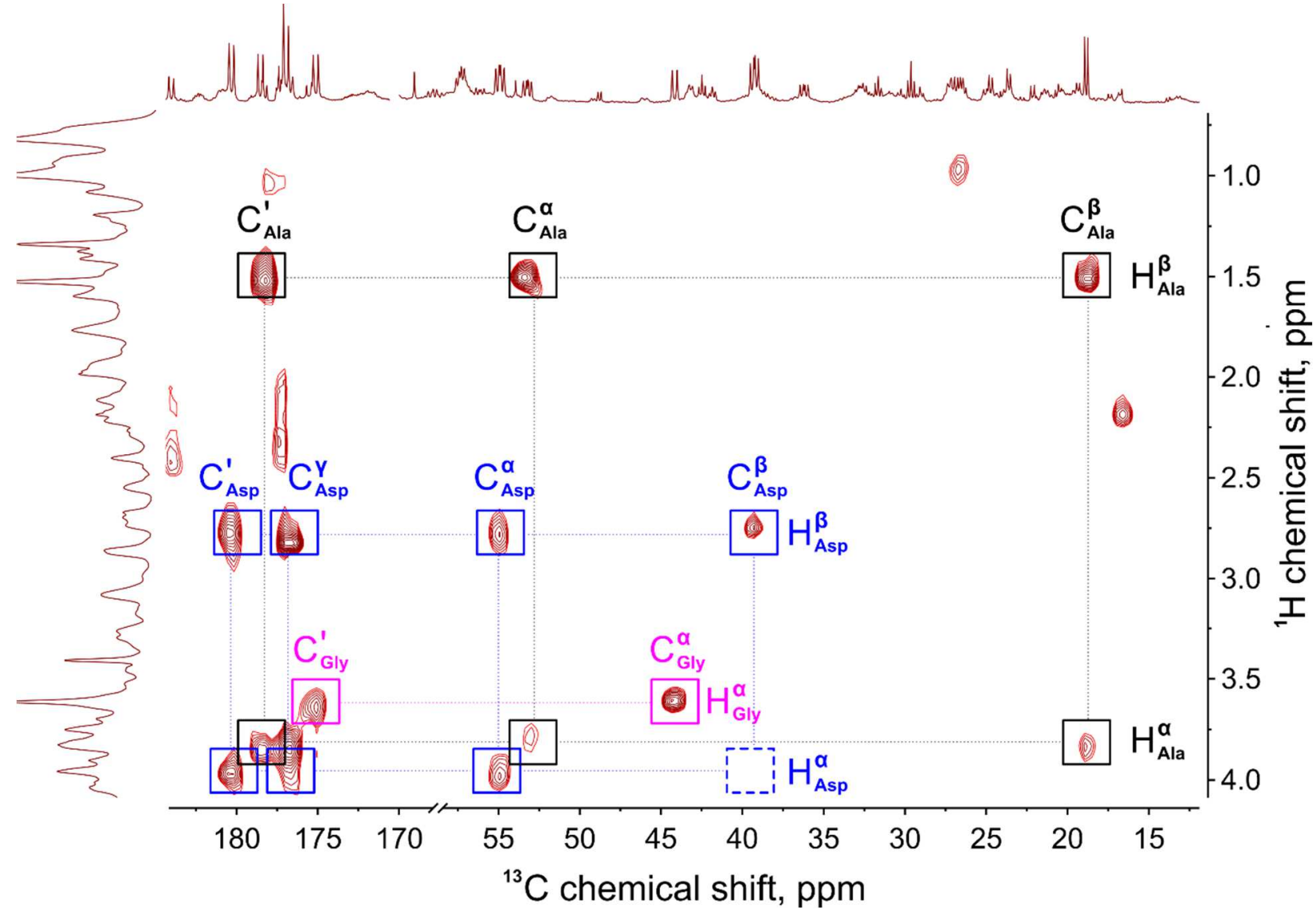

Figure 4. ZULF-TOCSY spectrum of the ISOGRO supernatant in $\mathrm{D}_{2} \mathrm{O}(\mathrm{pH}=6.5)$. Experimental parameters: $B_{U L}=50 \mathrm{nT}, t_{\text {mix }}=40$ ms, 64 transients in indirect direction, 960 scans per transient, relaxation delay $3 \mathrm{~s}$, total experiment duration ca. 69 hours.

ZULF-TOCSY spectra allow one to highlight heteronuclear coupling networks. A spectrum with all possible correlations provides invaluable information to identify individual molecules in complex mixtures. We have used ZULF-TOCSY to analyze a solution of the ISOGRO growth medium, which contains various isotopically labelled small biomolecules, e.g., amino acids. The ${ }^{1} \mathrm{H}^{13} \mathrm{CZULF}-\mathrm{TOSCY}$ spectrum of the ISOGRO sample (Figure 4) highlights the coupling networks in individual amino acids: signals of alanine, glycine and aspartate can be clearly identified. Hence, ZULF-TOCSY is a powerful method for the analysis of small molecules, whereas bigger molecules are expected to be subject to efficient relaxation during the field jumps $B_{0} \rightarrow B_{U L}$ and $B_{U L} \rightarrow B_{0}$, which reduces the signals. We expect that when $T_{1}$ is shorter than or comparable to $t_{s w}$ (which is the case for bigger molecules), ZULF-TOCSY peaks will be strongly reduced. This clear advantage of ZULF-TOCSY for the analysis of small molecules is also a limit of the method for its application to large-size molecules.

\section{Conclusion}

In this work, we introduce the use of isotropic spin mixing under ZULF-NMR conditions to develop new high-resolution 2D-NMR experiments. Specifically, we propose to utilize coherent polarization transfer among heteronuclear spin systems to implement an analogue of the widely used TOCSY experiment, which is applied to homonuclear spin systems. Such a ZULF-TOCSY experiment allows one to identify 
coupling networks of all NMR active nuclei, displayed in ${ }^{1} \mathrm{H}^{-13} \mathrm{C}$ or ${ }^{1} \mathrm{H}-{ }^{15} \mathrm{~N} 2 \mathrm{D}$ correlations. We illustrate the features of ZULF-TOCSY on two isotopically enriched amino acids, leucine and lysine. Furthermore, we demonstrate the efficiency of ZULF-TOCSY as an analytical tool for complex mixture on a solution of ISOGRO growth medium, which is a complex mixture of various biomolecules. ZULF-TOCSY enables quick identification of individual amino acids in the mixture by their coupled intramolecular spin networks.

Mixing at ultralow field can be used as a building block in multi-dimensional experiments. The simultaneous correlation of all active nuclei makes ZULF-TOCSY particularly suited for parallel detection. ${ }^{38}$ The method was demonstrated on isotopically labeled amino acids but can also be applied to molecules at natural abundance and is promising for the analysis of molecules containing NMR active nuclei, such as ${ }^{19} \mathrm{~F},{ }^{29} \mathrm{Si}$ or ${ }^{31} \mathrm{P}$. An advantage of the method is that the mixing time does not need to be set precisely to highlight specific spin-spin interactions: under ZULF-NMR conditions spin polarization is rapidly distributed over the entire coupled network. We anticipate that the proposed method can be used to assign NMR signals and to identify small molecules in complex mixtures as encountered in chemical synthesis, as well as mixtures of biomolecules and metabolites in biological fluids or lysed cells.

\section{Acknowledgements}

The Novosibirsk group acknowledges the Russian Science Foundation (grant agreement No. 19-43-04116) for supporting polarization transfer experiments at ultralow fields and the Russian Foundation for Basic Research (project number 19-33-90251) for supporting ZULF-TOCSY experiments. I.Z. acknowledges support from the French embassy in the Russian Federation, in the framework of the Ostrogradsky fellowship (project No. 933824A). G.B. gratefully acknowledges financial support by the Deutsche Forschungsgemeinschaft under contract Bu-911-29-1 in the framework of a joint DFG/RSF-project.

\section{References}

1. Ernst, R. R.; Bodenhausen, G.; Wokaun, A., Principles of Nuclear Magnetic Resonance in One and Two Dimensions. Clarendon Press ; Oxford University Press: Oxford Oxfordshire, New York, 1987.

2. Bax, A.; Freeman, R., Investigation of Complex Networks of Spin-Spin Coupling by TwoDimensional NMR. J. Magn. Reson. 1981, 44 (3), 542-561.

3. Bodenhausen, G.; Ruben, D. J., Natural Abundance N-15 NMR by Enhanced Heteronuclear Spectroscopy. Chem. Phys. Lett. 1980, 69 (1), 185-189.

4. Müller, L., Sensitivity Enhanced Detection of Weak Nuclei Using Heteronuclear Multiple Quantum Coherence. J. Am. Chem. Soc. 1979, 101 (16), 4481-4484.

5. Bax, A.; Summers, M. F., H-1 and C-13 Assignments from Sensitivity-Enhanced Detection of Heteronuclear Multiple-Bond Connectivity by 2D Multiple Quantum NMR. J. Am. Chem. Soc. 1986, 108 (8), 2093-2094.

6. Dingley, A. J.; Grzesiek, S., Direct Observation of Hydrogen Bonds in Nucleic Acid Base Pairs by Internucleotide ${ }^{2} J_{N N}$ Couplings. J. Am. Chem. Soc. 1998, 120 (33), 8293-8297.

7. Golubev, N. S.; Shenderovich, I. G.; Smirnov, S. N.; Denisov, G. S.; Limbach, H. H., Nuclear Scalar Spin-Spin Coupling Reveals Novel Properties of Low-Barrier Hydrogen Bonds in a Polar Environment. Chem. Eur. J. 1999, 5 (2), 492-497.

8. Cordier, F.; Rogowski, M.; Grzesiek, S.; Bax, A., Observation of through-Hydrogen-Bond ${ }^{2 \mathrm{~h}} \mathrm{JHC}_{\mathrm{H}}$ in a Perdeuterated Protein. J. Magn. Reson. 1999, 140 (2), 510-512.

9. Kumar, A.; Ernst, R. R.; Wuthrich, K., A Two-Dimensional Nuclear Overhauser Enhancement (2D NOE) Experiment for the Elucidation of Complete Proton-Proton Cross-Relaxation Networks in Biological Macromolecules. Biochem. Biophys. Res. Commun. 1980, 95 (1), 1-6.

10. Braunschweiler, L.; Ernst, R. R., Coherence Transfer by Isotropic Mixing - Application to Proton Correlation Spectroscopy. J. Magn. Reson. 1983, 53 (3), 521-528.

11. Cavanagh, J.; Fairbrother, W. J.; Palmer lii, A. G.; Rance, M.; Skelton, N. J., Chapter 6 - Experimental 1H NMR Methods. In Protein NMR Spectroscopy (Second Edition), Academic Press: Burlington, 2007; pp 405-532. 
12. Fiala, R.; Sklenár̆, V., NMR of Nucleic Acids. In Bionmr in Drug Research, R. Mannhold, H. K., G. Folkers and O. Zerbe, Ed. 2003; pp 121-146.

13. Cheng, H. N.; Neiss, T. G., Solution NMR Spectroscopy of Food Polysaccharides. Polym. Rev. (Philadelphia, PA, U. S.) 2012, 52 (2), 81-114.

14. Fan, W. M. T., Metabolite Profiling by One- and Two-Dimensional NMR Analysis of Complex Mixtures. Prog. Nucl. Magn. Reson. Spectrosc. 1996, 28, 161-219.

15. Morris, G. A.; Gibbs, A., Long-Range Heteronuclear Correlation 2D NMR by MLEV-16 Isotropic Mixing. Magn. Reson. Chem. 1991, 29 (1), 83-87.

16. Carlomagno, T.; Luy, B.; Glaser, S. J., "Kin" HEHAHA Sequences, Heteronuclear Hartmann-Hahn Transfer with Different Bandwidths for Spins I and S. J. Magn. Reson. 1997, 126 (1), 110-119.

17. Parella, T.; Espinosa, J. F., Long-Range Proton-Carbon Coupling Constants: NMR Methods and Applications. Prog. Nucl. Magn. Reson. Spectrosc. 2013, 73, 17-55.

18. Cavanagh, J.; Fairbrother, W. J.; Palmer, A. G.; Rance, M.; Skelton, N. J., Chapter 7 - Heteronuclear NMR Experiments. In Protein NMR Spectroscopy (Second Edition), Cavanagh, J.; Fairbrother, W. J.; Palmer, A. G.; Rance, M.; Skelton, N. J., Eds. Academic Press: Burlington, 2007; pp 533-678.

19. Marion, D.; Driscoll, P. C.; Kay, L. E.; Wingfield, P. T.; Bax, A.; Gronenborn, A. M.; Clore, G. M., Overcoming the Overlap Problem in the Assignment of Proton NMR Spectra of Larger Proteins by Use of Three-Dimensional Heteronuclear Proton-Nitrogen-15 Hartmann-Hahn-Multiple Quantum Coherence and Nuclear Overhauser-Multiple Quantum Coherence Spectroscopy: Application to Interleukin 1.beta. Biochemistry 1989, 28 (15), 6150-6156.

20. Blanchard, J. W.; Budker, D., Zero- to Ultralow-Field NMR. Emagres 2016, 5 (3), 1395-1409.

21. Redfield, A. G., Shuttling Device for High-Resolution Measurements of Relaxation and Related Phenomena in Solution at Low Field, Using a Shared Commercial $500 \mathrm{MHz}$ NMR Instrument. Magn. Reson. Chem. 2003, 41 (10), 753-768.

22. Grosse, S.; Gubaydullin, F.; Scheelken, H.; Vieth, H.-M.; Yurkovskaya, A. V., Field Cycling by Fast NMR Probe Transfer: Design and Application in Field-Dependent CIDNP Experiments. Appl. Magn. Reson. 1999, $17(2-3), 211-225$.

23. Cousin, S. F.; Charlier, C.; Kaderavek, P.; Marquardsen, T.; Tyburn, J. M.; Bovier, P. A.; Ulzega, S.; Speck, T.; Wilhelm, D.; Engelke, F.; Maas, W.; Sakellariou, D.; Bodenhausen, G.; Pelupessy, P.; Ferrage, F., High-Resolution Two-Field Nuclear Magnetic Resonance Spectroscopy. Phys. Chem. Chem. Phys. 2016, 18 (48), 33187-33194.

24. Ivanov, D.; Redfield, A. G., Field-Cycling Method with Central Transition Readout for Pure Quadrupole Resonance Detection in Dilute Systems. J. Magn. Reson. 2004, 166 (1), 19-27.

25. Merck Description of Isogro Sample. https://www.sigmaaldrich.com/technicaldocuments/articles/stable-isotopes/isogro-complex-growth-media.html (accessed 03.06.2020).

26. Zhukov, I. V.; Kiryutin, A. S.; Yurkovskaya, A. V.; Grishin, Y. A.; Vieth, H.-M.; Ivanov, K. L., FieldCycling NMR Experiments in Ultra-Wide Magnetic Field Range: Relaxation and Coherent Polarization Transfer. Phys. Chem. Chem. Phys. 2018, 20 (18), 12396-12405.

27. Jacobsen, N. E., NMR Spectroscopy Explained: Simplified Theory, Applications and Examples for Organic Chemistry and Structural Biology. NMR Spectroscopy Explained: Simplified Theory, Applications and Examples for Organic Chemistry and Structural Biology 2007, 1-668.

28. Wishart, D. S.; Bigam, C. G.; Yao, J.; Abildgaard, F.; Dyson, H. J.; Oldfield, E.; Markley, J. L.; Sykes, B. D., H-1, C-13 and N-15 Chemical Shift Referencing in Biomolecular NMR. J. Biomol. NMR 1995, 6 (2), 135-140.

29. Bernarding, J.; Buntkowsky, G.; Macholl, S.; Hartwig, S.; Burghoff, M.; Trahms, L., J-Coupling Nuclear Magnetic Resonance Spectroscopy of Liquids in Nt Fields. J. Am. Chem. Soc. 2006, 128 (3), 714715 .

30. Sjolander, T. F.; Tayler, M. C. D.; Kentner, A.; Budker, D.; Pines, A., 13C-Decoupled J-Coupling Spectroscopy Using Two-Dimensional Nuclear Magnetic Resonance at Zero-Field. J. Phys. Chem. Lett. 2017, 8 (7), 1512-1516.

31. Chou, C. Y.; Chu, M. L.; Chang, C. F.; Huang, T. H., A Compact High-Speed Mechanical Sample Shuttle for Field-Dependent High-Resolution Solution NMR. J. Magn. Reson. 2012, 214, 302-308. 
32. Ledbetter, M. P.; Theis, T.; Blanchard, J. W.; Ring, H.; Ganssle, P.; Appelt, S.; Blümich, B.; Pines, A.; Budker, D., Near-Zero-Field Nuclear Magnetic Resonance. Phys. Rev. Lett. 2011, 107 (10), 107601.

33. Ledbetter, M. P.; Crawford, C. W.; Pines, A.; Wemmer, D. E.; Knappe, S.; Kitching, J.; Budker, D., Optical Detection of NMR J-Spectra at Zero Magnetic Field. J. Magn. Reson. 2009, 199 (1), 25-29.

34. Budker, D.; Romalis, M., Optical Magnetometry. Nat. Phys. 2007, 3 (4), 227-234.

35. Barskiy, D. A.; Knecht, S.; Yurkovskaya, A. V.; Ivanov, K. L., Sabre: Chemical Kinetics and Spin Dynamics of the Formation of Hyperpolarization. Prog. Nucl. Magn. Reson. Spectrosc. 2019, 114-115, 3370.

36. Ivanov, K. L.; Yurkovskaya, A. V.; Vieth, H.-M., Coherent Transfer of Hyperpolarization in Coupled Spin Systems at Variable Magnetic Field. J. Chem. Phys. 2008, 128 (15), 154701(1-13).

37. Zhu, G.; Bax, A., Improved Linear Prediction for Truncated Signals of Known Phase. Journal of Magnetic Resonance (1969) 1990, 90 (2), 405-410.

38. Kupče, E. .; Freeman, R.; John, B. K., Parallel Acquisition of Two-Dimensional NMR Spectra of Several Nuclear Species. J. Am. Chem. Soc. 2006, 128 (30), 9606-9607. 


\title{
Supporting Information
}

for the article

\section{Total correlation spectroscopy across all NMR-active nuclei by mixing at zero field}

\author{
Ivan V. Zhukov ${ }^{a, b}$, Alexey S. Kiryutin ${ }^{a, b}$, Fabien Ferrage ${ }^{c}$, Gerd Buntkowsky ${ }^{d}$, \\ Alexandra V. Yurkovskayaa,b, Konstantin L. Ivanova,b, ${ }^{\text {* }}$ \\ a International Tomography Center SB RAS, Novosibirsk 630090, Russia \\ ${ }^{b}$ Novosibirsk State University, Novosibirsk 630090, Russia \\ c Laboratoire des Biomolécules, LBM, Département de chimie, École normale supérieure, PSL \\ University, Sorbonne Université, CNRS, 75005 Paris, France \\ ${ }^{d}$ Technical University of Darmstadt, Institute of Inorganic and Physical Chemistry, D-64287 \\ Darmstadt, Germany \\ * Corresponding author, email: ivanov@tomo.nsc.ru
}

\section{Contents}

1. Polarization transfer experiments under ZULF-NMR conditions.

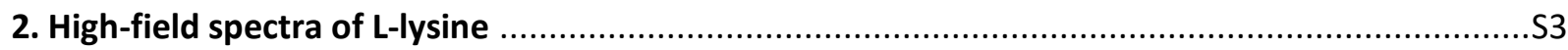

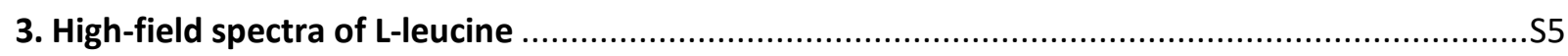

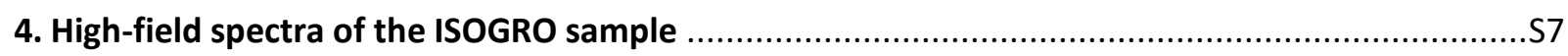




\section{Polarization transfer experiments under ZULF-NMR conditions}

In this subsection, we present optimization of the conditions for polarization transfer. Such experiments were performed according to the protocol shown in Figure 2 of the main article. In addition to ${ }^{1} \mathrm{H}-{ }^{13} \mathrm{C}$ polarization transfer experiments in isotopically labelled L-lysine, we studied the ${ }^{1} \mathrm{H}-{ }^{15} \mathrm{~N}$ transfer and performed the same experiments for the second amino acid, L-leucine.

The results for ${ }^{1} \mathrm{H}-{ }^{15} \mathrm{~N}$ transfer in L-lysine under ZULF-NMR conditions are shown in Figure S1. We have not observed pronounced oscillations in the dependence of polarization on the mixing time $t_{m i x}$ at an ultralow field $B_{U L}$. Nonetheless, we can demonstrate that polarization transfer takes place, as it is seen from the enhanced NMR signal of the ${ }^{15} \mathrm{~N}$ nuclei, which is higher than the thermal signal due to the transfer of the much higher polarization from protons. The results for the other amino acid, ${ }^{13} \mathrm{C},{ }^{15} \mathrm{~N}$-labeled Lleucine, are qualitatively similar. In both cases, the $t_{\text {mix }}$ times for the ${ }^{1} \mathrm{H} \rightarrow{ }^{15} \mathrm{~N}$ polarization transfer are about $50 \mathrm{~ms}$.

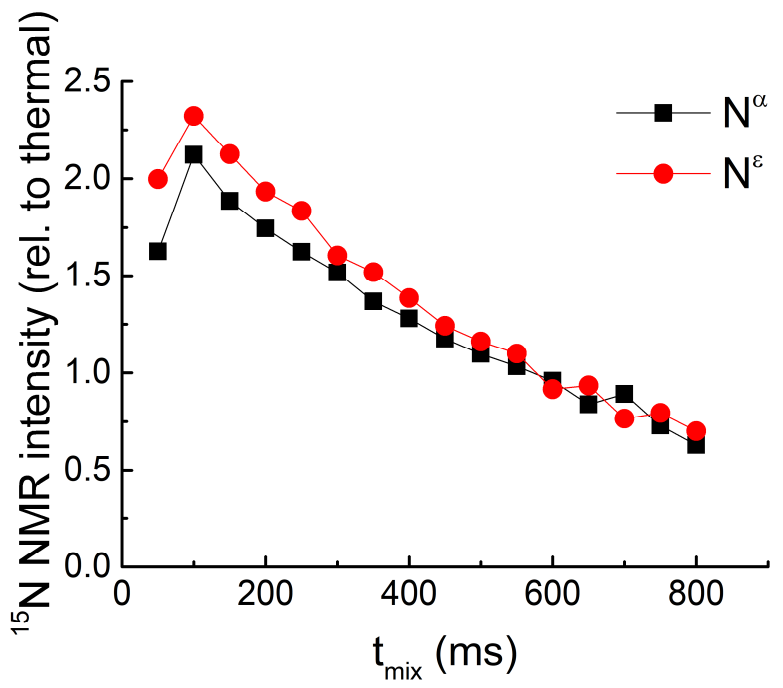

Figure S1. Dependence of ${ }^{15} \mathrm{~N} \mathrm{NMR}$ signal intensities of uniformly ${ }^{13} \mathrm{C},{ }^{15} \mathrm{~N}$-labeled L-lysine on the mixing time $t_{\text {mix }}$ at an ultralow field, here $B_{U L}=100 \mathrm{nT}$. The signal intensity is normalized to that of the signal at $B=B_{0}$ at equilibrium conditions.

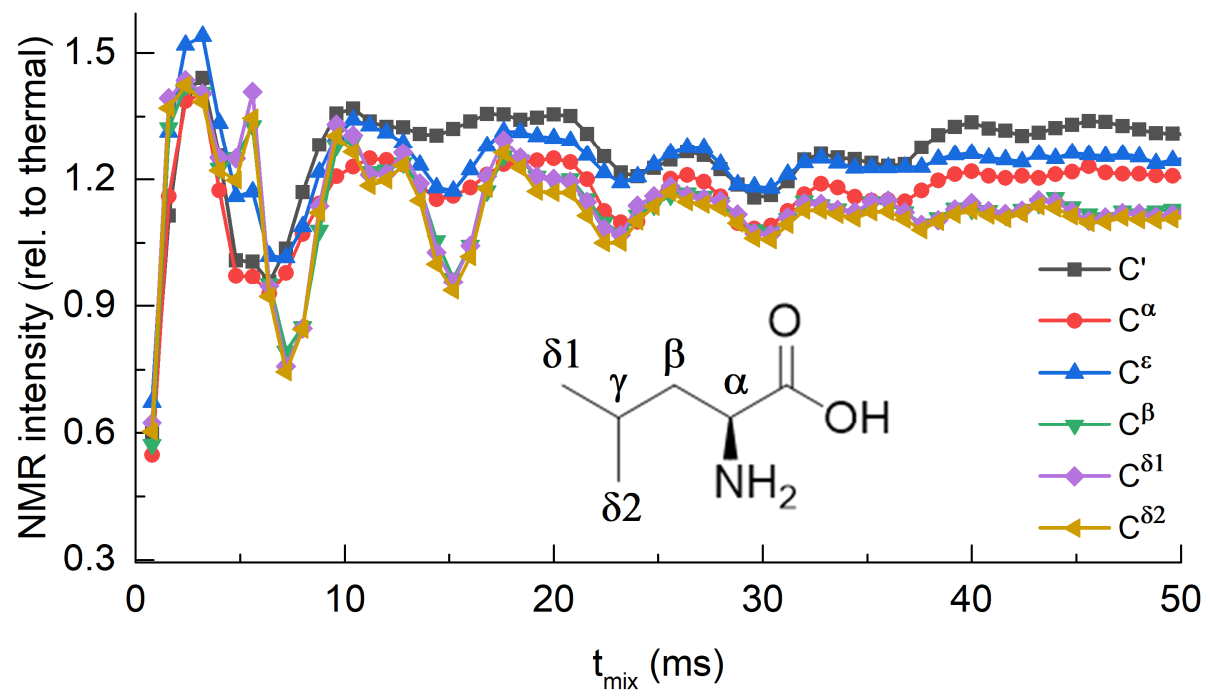

Figure S2. Dependence of ${ }^{13} \mathrm{C}$ NMR signal intensities of uniformly ${ }^{13} \mathrm{C},{ }^{15} \mathrm{~N}$-labeled L-leucine on $t_{\text {mix }}$. The resulting signals are normalized to the thermal signals at $B=B_{0}$. 
On the other hand, for the transfer from protons to ${ }^{13} \mathrm{C}$ nuclei we always seen pronounced quantum oscillations, both for L-lysine (see Figure 2) and for L-leucine (see Figure S2). The optimal mixing time can then be evaluated as approximately $3 \mathrm{~ms}$.

Having optimized conditions for polarization transfer, we are able to record ZULF-TOCSY spectra, which are shown in the main article for L-lysine (see Figure 3) and for L-leucine (see Figure S3). In L-leucine, we can observe all possible cross-peaks in the ${ }^{1} \mathrm{H}^{-13} \mathrm{C}$ and ${ }^{1} \mathrm{H}-{ }^{15} \mathrm{~N}$ ZULF-TOCSY spectra: each carbon exhibits a cross-peak with each proton and the ${ }^{15} \mathrm{~N}$ spin also has cross-peaks with all protons in the molecule.
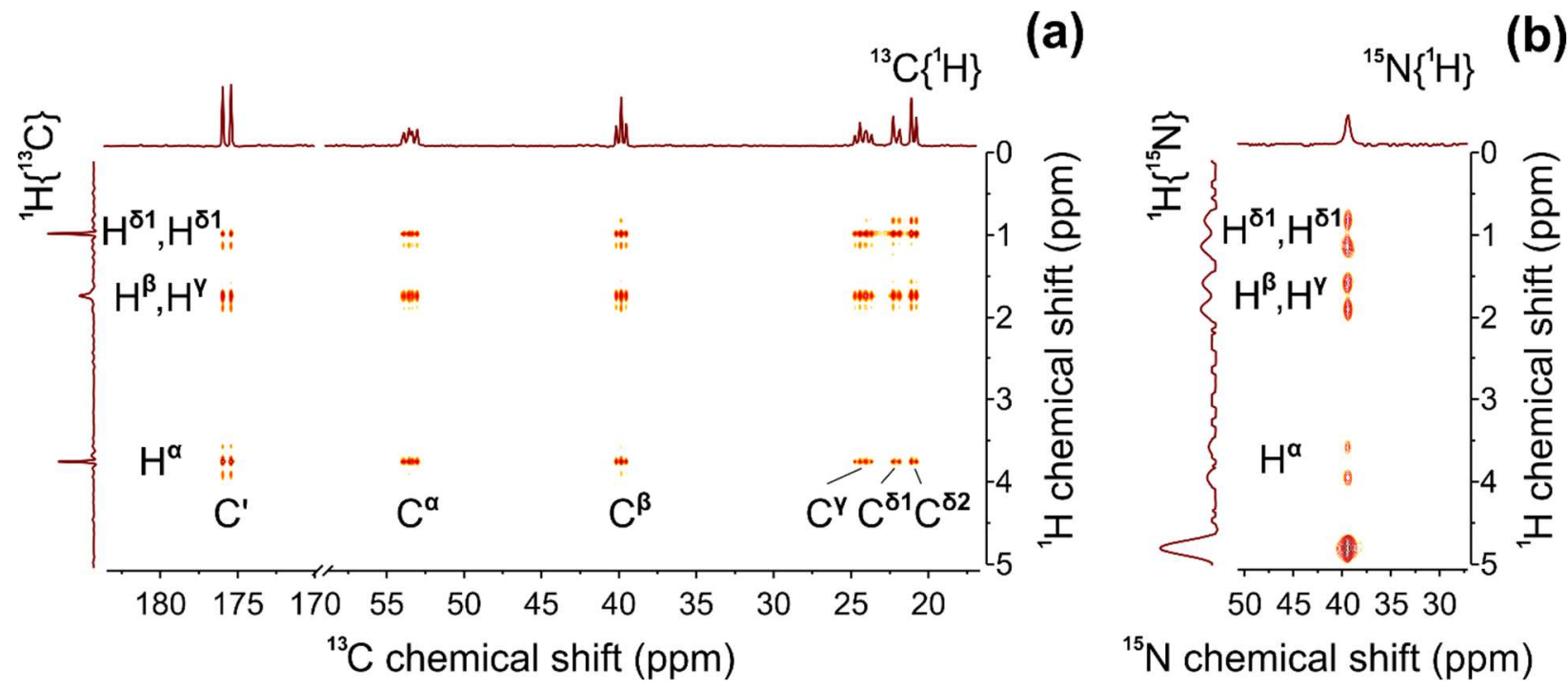

Figure S3. (a) ${ }^{13} \mathrm{C}-{ }^{1} \mathrm{H}$ ZULF-TOCSY spectrum of $100 \mathrm{mM}$ uniformly ${ }^{13} \mathrm{C}^{15} \mathrm{~N}$-labeled L-leucine in $\mathrm{D}_{2} \mathrm{O}$. Experimental parameters: $B_{U L}=100 \mathrm{nT}, t_{\text {mix }}=3 \mathrm{~ms}, 128$ transients per $5 \mathrm{ppm}$ (resolution $31.3 \mathrm{~Hz}$ ) in indirect direction, 4 scans per transient, relaxation delay $5 \mathrm{~s}$, total experiment duration ca. 70 minutes. Linear prediction of indirect dimension FID points 129-512 was applied using Zhu-Bax method. Acquired data multiplied by $90^{\circ}$ shifted squared sinusoidal window function in both dimensions. Spectrum presented by $2048 \times 512$ data points. (b) ${ }^{15} \mathrm{~N}-{ }^{1} \mathrm{H}$ ZULF-TOCSY spectrum of $100 \mathrm{mM}{ }^{13} \mathrm{C}_{1}^{15} \mathrm{~N}$ uniformly labeled L-leucine in $\mathrm{D}_{2} \mathrm{O}$. Experimental parameters: $B_{U L}=100 \mathrm{nT}, t_{\text {mix }}=50 \mathrm{~ms}, 64$ transients per $6 \mathrm{ppm}$ (resolution $75 \mathrm{~Hz}$ ) in indirect direction, 128 scans per transient, relaxation delay $25 \mathrm{~s}$, total experiment duration ca. 62 hours. Linear prediction of indirect dimension FID points 64-256 was applied using the Zhu-Bax method. Acquired data multiplied by $90^{\circ}$ shifted squared sinusoidal window function in both dimensions. Spectrum presented by $2048 \times 256$ data points. Splitting in the indirect dimension is due to a non-refocused scalar coupling with the ${ }^{13} \mathrm{C}$ nuclei directly bound to a proton. 


\section{High-field spectra of L-lysine}

In this section, we present the high-field NMR spectra of L-lysine, namely the $1 \mathrm{D}$-spectra for ${ }^{1} \mathrm{H},{ }^{13} \mathrm{C}$ and ${ }^{15} \mathrm{~N}$ nuclei and 2D-spectra, such as ${ }^{1} \mathrm{H}_{-}{ }^{13} \mathrm{C}$ HSQC, ${ }^{1} \mathrm{H}_{-}{ }^{15} \mathrm{C} \mathrm{HSQC},{ }^{1} \mathrm{H}-{ }^{13} \mathrm{C} \mathrm{HMBC}$. For the 2D-spectra, experimental parameters, dealing with data acquisition, are given in figure legends.

In the 1D-spectra we can resolve and assign the signals of all nuclei of interest, see Figure S5. In the case of ${ }^{13} \mathrm{C}$ and ${ }^{15} \mathrm{~N}$, the spectra have been acquired with proton decoupling. Hence, the signals in this spectra exhibit multiplet structure originating from the homonuclear ${ }^{13} \mathrm{C}-{ }^{13} \mathrm{C}$ and heteronuclear ${ }^{13} \mathrm{C}-{ }^{15} \mathrm{~N}$ scalar couplings.

(a)

(b)
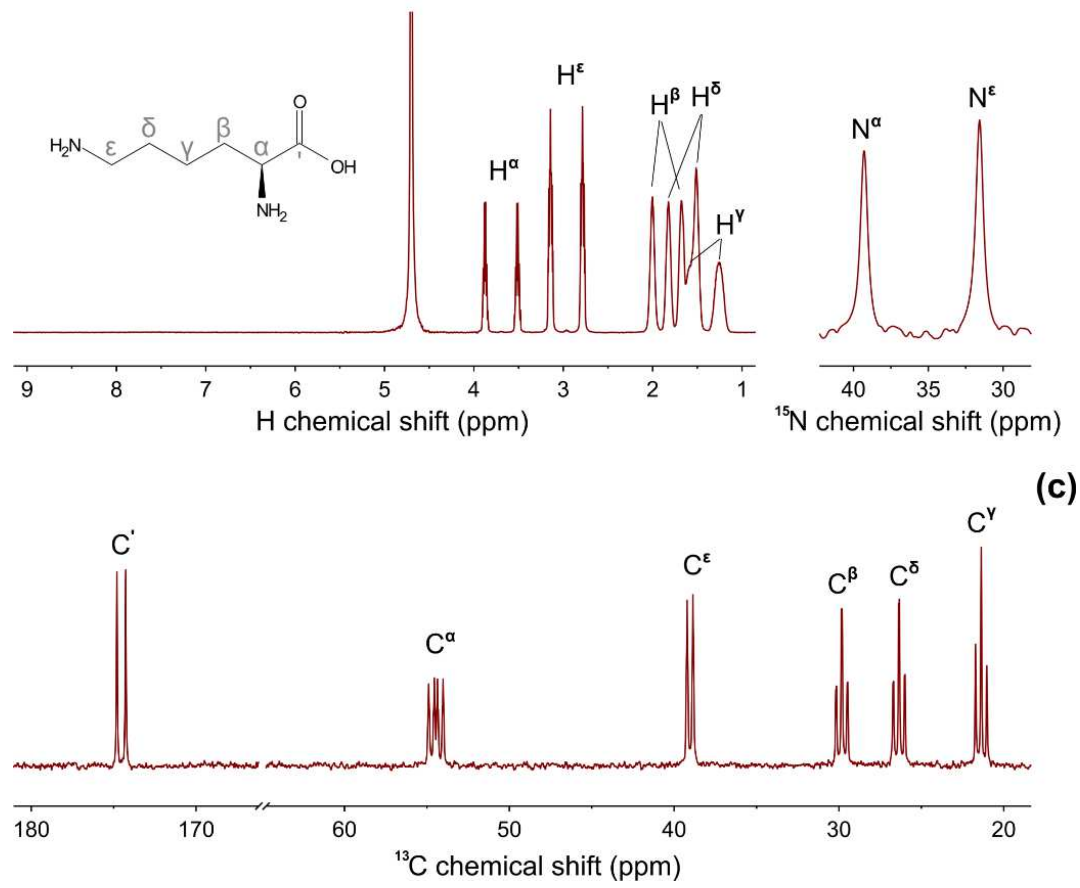

(c)

Figure S5. 1D NMR spectra of $100 \mathrm{mM}{ }^{13} \mathrm{C},{ }^{15} \mathrm{~N}$ uniformly labeled L-lysine in D2O. (a) $400 \mathrm{MHz}{ }^{1} \mathrm{H}$ NMR spectrum. (b) $40.55 \mathrm{MHz}{ }^{15} \mathrm{~N}$ NMR spectrum acquired with ${ }^{1} \mathrm{H}$ decoupling. (c) $100.62 \mathrm{MHz}{ }^{13} \mathrm{C} \mathrm{NMR}$ spectrum acquired with ${ }^{1} \mathrm{H}$ decoupling.

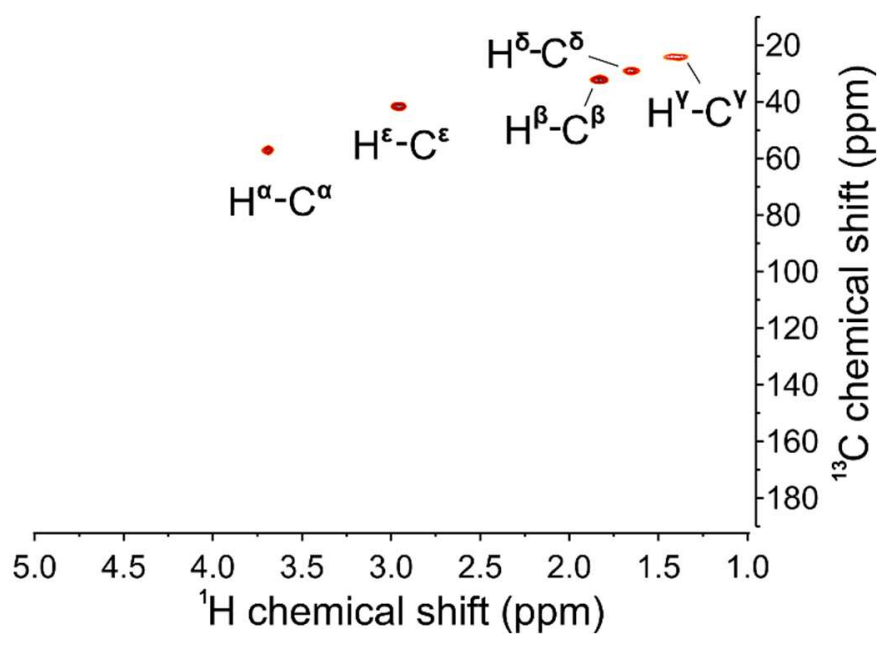

Figure S6. $400 \mathrm{MHz}{ }^{1} \mathrm{H}-{ }^{13} \mathrm{C}$ HSQC NMR spectrum of $100 \mathrm{mM}{ }^{13} \mathrm{C}_{1}^{15} \mathrm{~N}$ uniformly labeled L-lysine in $\mathrm{D}_{2} \mathrm{O}$. The indirect dimension is sampled with 256 transients over the $180 \mathrm{ppm}$ spectral width, leading to approximately $140 \mathrm{~Hz}$ per point spectral resolution. Duration of the mixing period is $7 \mathrm{~ms}$. The acquisition time was ca. $15 \mathrm{~min}$. Acquired signals were multiplied by $90^{\circ}$ shifted squared sinusoidal $\left(\operatorname{Sin}^{2}\right)$ window function in both dimensions. The spectrum contains $256 \times 256$ data points. 
To gain insight into heteronuclear polarization transfer pathways and efficiency we have acquired HSQC and $\mathrm{HMBC}$ spectra.

In all spectra, we were able to see a limited number of cross-peaks. For instance, in the ${ }^{1} \mathrm{H}-{ }^{13} \mathrm{C} \mathrm{HSQC}$ spectrum we can observe only the cross-peaks between the nearest neighbors, see Figure $\mathrm{S6}$. In the ${ }^{1} \mathrm{H}$ ${ }^{13} \mathrm{CHSQC}$ spectrum, see Figure S7, we see the following cross-peaks: $\mathrm{N} \varepsilon-\mathrm{H} \varepsilon, \mathrm{N} \varepsilon-\mathrm{H} \delta, \mathrm{N} \alpha-\mathrm{H} \beta$. Hence, in some cases, transfer to more remote protons is feasible, but uniform transfer, like in ZULF-TOCSY, is never observed.

We also acquired the ${ }^{1} \mathrm{H}-{ }^{13} \mathrm{C} \mathrm{HMBC}$ spectrum, see Figure $\mathrm{S} 8$, to analyze polarization transfer further. Like in the HSQC case, we are able to observe only a limited number of cross-peaks, which is in contrast with the ZULF-TOCSY spectra.

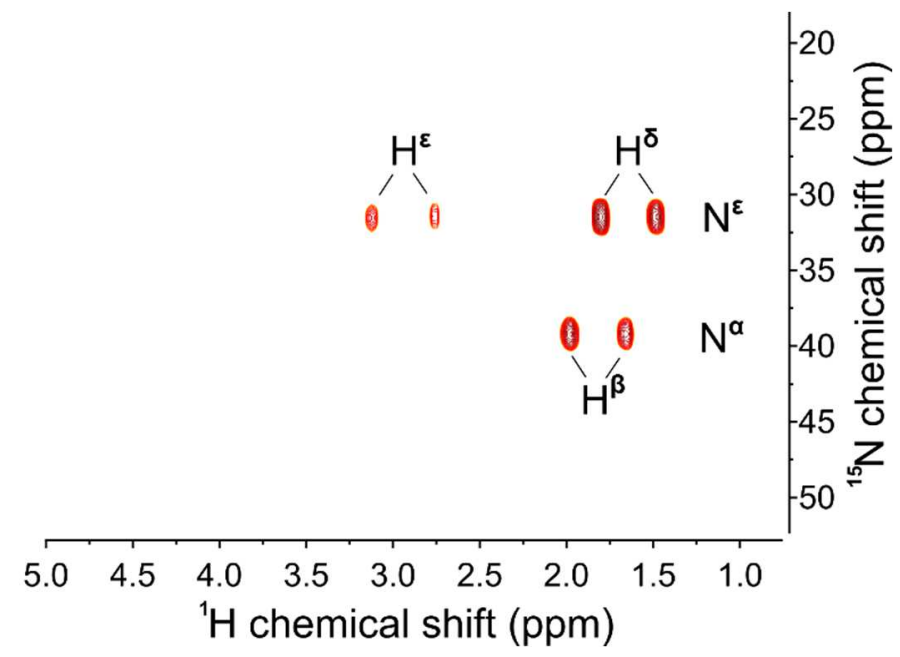

Figure S7. $400 \mathrm{MHz}{ }^{1} \mathrm{H}-{ }^{15} \mathrm{~N}$ HSQC NMR spectrum of $100 \mathrm{mM}{ }^{13} \mathrm{C}_{1}^{15} \mathrm{~N}$ uniformly labeled L-lysine in $\mathrm{D}_{2} \mathrm{O} .{ }^{1} \mathrm{H}_{-}{ }^{13} \mathrm{C}$ scalar couplings cause cross-peak splitting in the proton frequency axis. The indirect dimension was sampled with 64 transients over the $46 \mathrm{ppm}$ spectral window, leading to approximately $50 \mathrm{~Hz}$ spectral resolution. Duration of the mixing period is $125.2 \mathrm{~ms}$. The acquisition time was ca. $5 \mathrm{~min}$. Acquired signals were multiplied by $90^{\circ}$ shifted squared sinusoidal window function in both dimensions. The spectrum contains by $1024 \times 256$ data points.

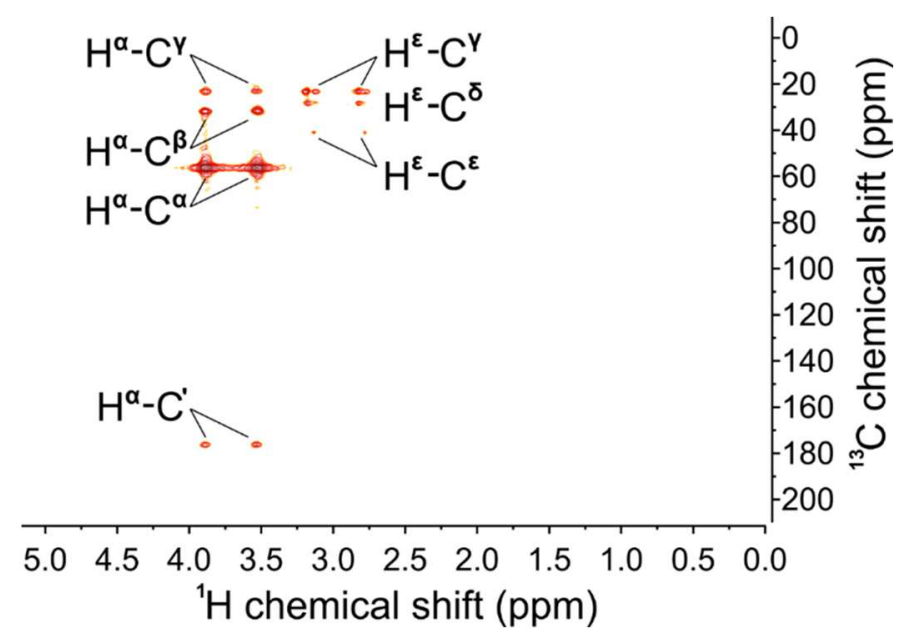

Figure S8. $400 \mathrm{MHz}{ }^{1} \mathrm{H}_{-}{ }^{13} \mathrm{C}$ HMBC spectrum of $100 \mathrm{mM}{ }^{13} \mathrm{C}_{1}^{15} \mathrm{~N}$ uniformly labeled L-lysine in $\mathrm{D}_{2} \mathrm{O}$. To record the spectrum Bruker's build-in pulse program "hmbcgplpndqf" was used, with J-filter cutting off couplings below $3 \mathrm{~Hz}$ or higher than $135 \mathrm{~Hz}$. The indirect dimension was sampled with 256 transients over the 220 ppm spectral window, leading to approximately $170 \mathrm{~Hz}$ spectral resolution. Duration of the mixing period is $171 \mathrm{~ms}$. The acquisition time was ca. $30 \mathrm{~min}$. The acquired signals were multiplied by $90^{\circ}$ shifted squared sinusoidal window function in both dimensions. The spectrum contains $512 \times 512$ data points. 


\section{High-field spectra of L-leucine}

We have also performed a similar analysis for L-lysine. In this section, we present the 1D-spectra for ${ }^{1} \mathrm{H}$, ${ }^{13} \mathrm{C}$ and ${ }^{15} \mathrm{~N}$ nuclei and 2D-spectra of interest, namely, ${ }^{1} \mathrm{H}-{ }^{13} \mathrm{C} \mathrm{HSQC},{ }^{1} \mathrm{H}^{-15} \mathrm{C} \mathrm{HSOC},{ }^{1} \mathrm{H}^{-13} \mathrm{C} \mathrm{HMBC}$. For the 2Dspectra, experimental parameters, dealing with data acquisition, are given in figure legends.

In the 1D-spectra, all signals of interest are well resolved and can be assigned, see Figure S5. In the case of ${ }^{13} \mathrm{C}$ and ${ }^{15} \mathrm{~N}$, the spectra have been acquired with proton decoupling (the multiplet structure in these spectra is coming from the homonuclear ${ }^{13} \mathrm{C}-{ }^{13} \mathrm{C}$ and heteronuclear ${ }^{13} \mathrm{C}-{ }^{15} \mathrm{~N}$ scalar couplings).

(a)

(b)
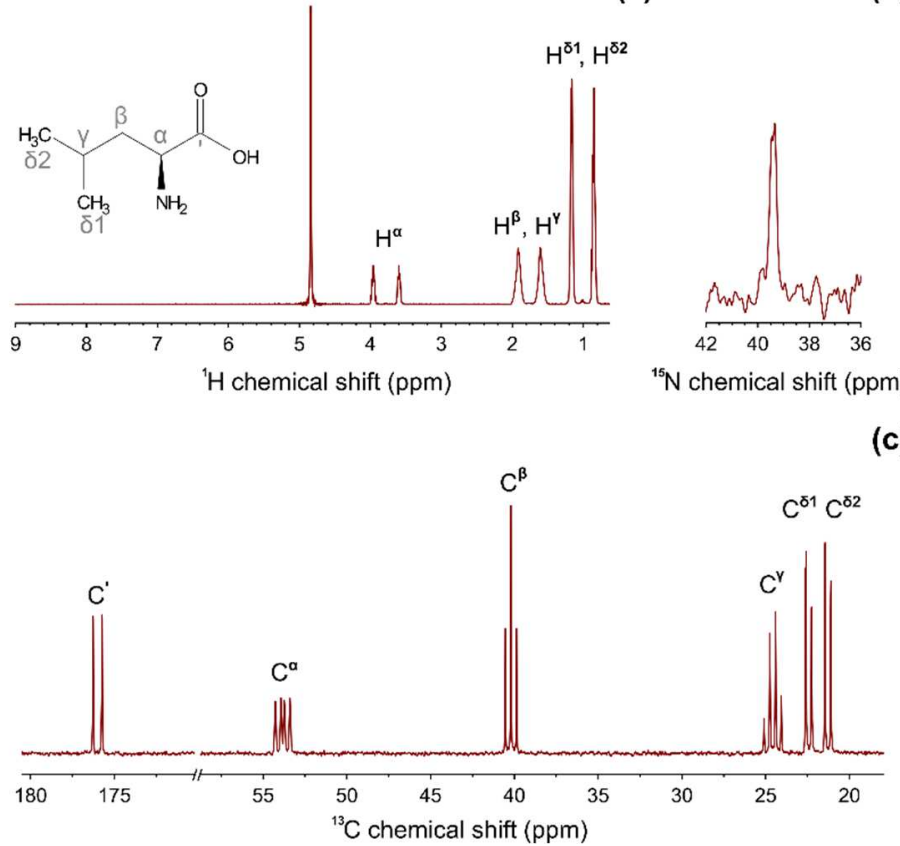

Figure S9. 1D NMR spectra of $100 \mathrm{mM}{ }^{13} \mathrm{C},{ }^{15} \mathrm{~N}$ uniformly labeled L-leucine in $\mathrm{D}_{2} \mathrm{O}$. (a) $400 \mathrm{MHz}{ }^{1} \mathrm{H}$ NMR spectrum. (b) $40.55 \mathrm{MHz}{ }^{15} \mathrm{~N}$ NMR spectrum acquired with ${ }^{1} \mathrm{H}$ decoupling. (c) $100.62 \mathrm{MHz}{ }^{13} \mathrm{C}$ NMR spectrum acquired with ${ }^{1} \mathrm{H}$ decoupling.

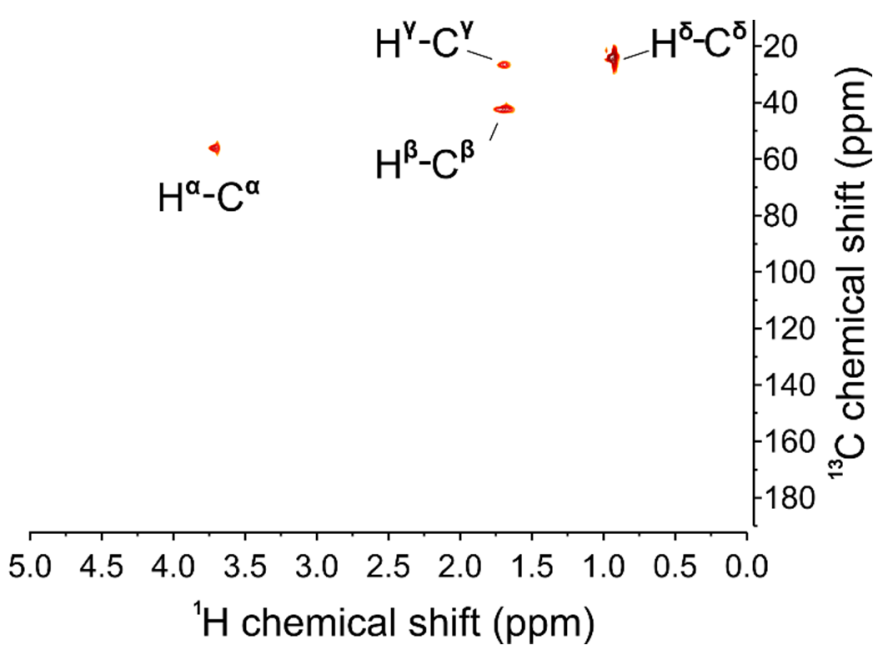

Figure S10. $400 \mathrm{MHz}{ }^{1} \mathrm{H}^{13} \mathrm{C}$ HSQC NMR spectrum of $100 \mathrm{mM}{ }^{13} \mathrm{C}^{15} \mathrm{~N}$ uniformly labeled L-leucine in $\mathrm{D}_{2} \mathrm{O}$. The parameters for data acquisition are exactly the same as those for the ${ }^{1} \mathrm{H}_{-}{ }^{13} \mathrm{C}$ HSQC spectrum of L-lysine, see Figure S6.

Like in the case of L-lysine, all 2D-spectra exhibit only a limited number of cross-peaks: this is true for ${ }^{1} \mathrm{H}$ ${ }^{13} \mathrm{C} \mathrm{HSQC}$ (Figure S10), ${ }^{1} \mathrm{H}_{-}{ }^{15} \mathrm{~N} \mathrm{HSQC}$ (Figure S11) and ${ }^{1} \mathrm{H}_{-}{ }^{13} \mathrm{C} \mathrm{HMBC}$ (Figure S12). In the ${ }^{1} \mathrm{H}_{-}{ }^{13} \mathrm{C} \mathrm{HSQC}$ crosspeaks are found only between the nearest neighbors in the molecule; in the ${ }^{1} \mathrm{H}^{15}{ }^{15} \mathrm{~N}$ HSQC spectrum polarization transfer between the nitrogen nucleus and only two protons can be seen. In the ${ }^{1} \mathrm{H}_{-}{ }^{13} \mathrm{C} \mathrm{HMBC}$ 
spectrum addition cross-peaks are visible, but distribution of polarization over the entire molecule is not possible.

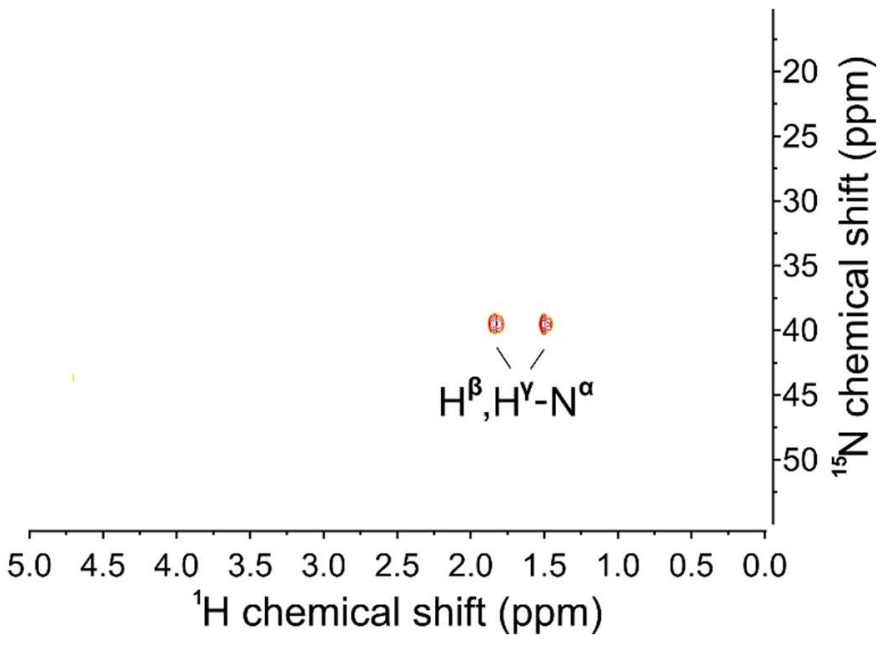

Figure S11. $400 \mathrm{MHz}{ }^{1} \mathrm{H}_{-}{ }^{15} \mathrm{~N}$ HSQC NMR spectra of $100 \mathrm{mM}{ }^{13} \mathrm{C}_{1}^{15} \mathrm{~N}$ uniformly labelled L-leucine in $\mathrm{D}_{2} \mathrm{O}$. The parameters for data acquisition are exactly the same as those for the ${ }^{1} \mathrm{H}-{ }^{15} \mathrm{~N} \mathrm{HSQC}$ spectrum of L-lysine, see Figure S7.

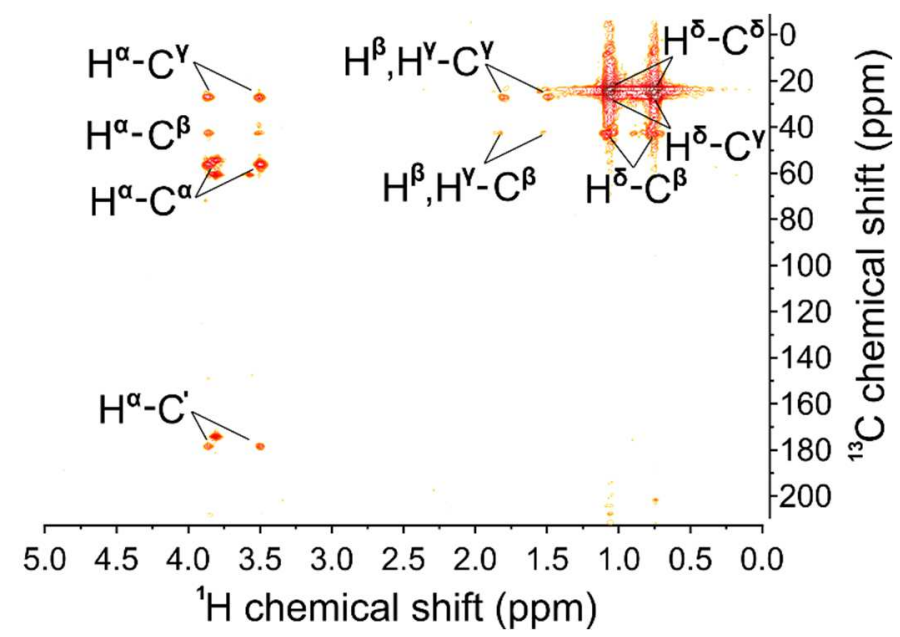

Figure S12. $400 \mathrm{MHz}{ }^{1} \mathrm{H}_{-}{ }^{13} \mathrm{C}$ HMBC NMR spectra of $100 \mathrm{mM}{ }^{13} \mathrm{C}_{,}^{15} \mathrm{~N}$ uniformly labelled L-leucine in $\mathrm{D}_{2} \mathrm{O}$. The parameters for data acquisition are exactly the same as those for the ${ }^{1} \mathrm{H}-{ }^{15} \mathrm{~N}$ HSQC spectrum of L-lysine, see Figure S7, except for the number of points in the spectrum, which contains $256 \times 512$ data points. 


\section{High-field spectra of the ISOGRO sample}

In this section, we present the data for the ISOGRO sample. 1D-spectra for ${ }^{1} \mathrm{H}$ and ${ }^{13} \mathrm{C}$ nuclei (acquired at $B_{0}=16.4$ tesla) are shown in Figures S13 and S14, respectively. In both cases, we obtain crowded spectra with many NMR lines. Except for the residual HDO signal the most intense signals at Figure S13 are glycine $\mathrm{H}^{\alpha}$ (doublet centered at $3.6 \mathrm{ppm}$ ) and methyl protons of alanine (doublet at $1.6 \mathrm{ppm}$ ) and leucine (doublet at $1.06 \mathrm{ppm})$. In the ${ }^{13} \mathrm{C}$ NMR spectra shown in Figure S14 one can additionally recognize signals of aromatic carbon nuclei of tyrosine (at 133.5 and $118.6 \mathrm{ppm}$ ) and tryptophan (around $130 \mathrm{ppm}$ ) as well as glycose signals in 70-100 ppm region. Assignment of other signals is not straightforward, requiring acquisition of the ${ }^{1} \mathrm{H}-{ }^{13} \mathrm{C}$ HSQC (Figure S15) and ${ }^{1} \mathrm{H}^{-13} \mathrm{C} \mathrm{HMBC}$ (Figure S16) spectra.

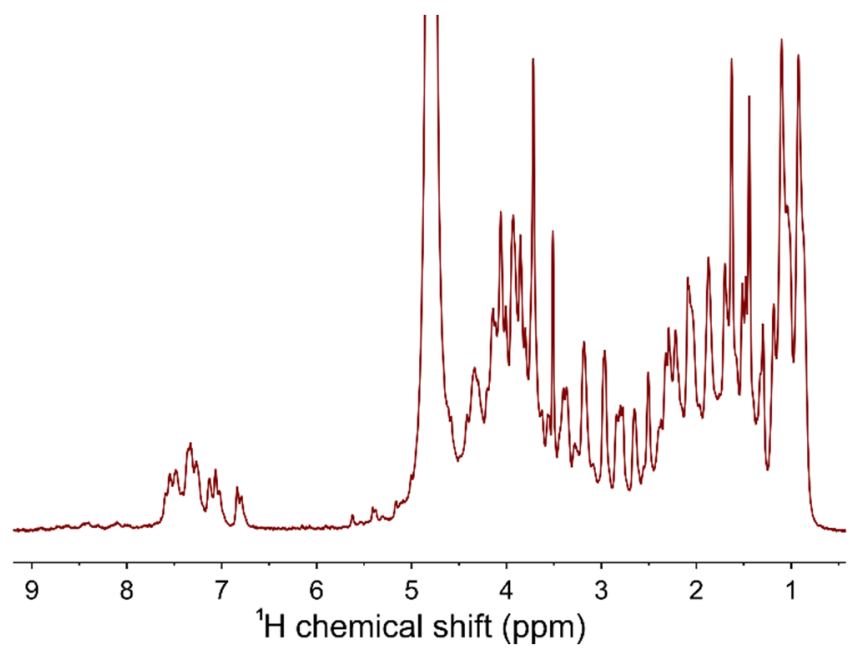

Figure S13. $700 \mathrm{MHz}{ }^{1} \mathrm{H}$ NMR spectrum of the ISOGRO supernatant in $\mathrm{D}_{2} \mathrm{O}(\mathrm{pH} 6.5)$.

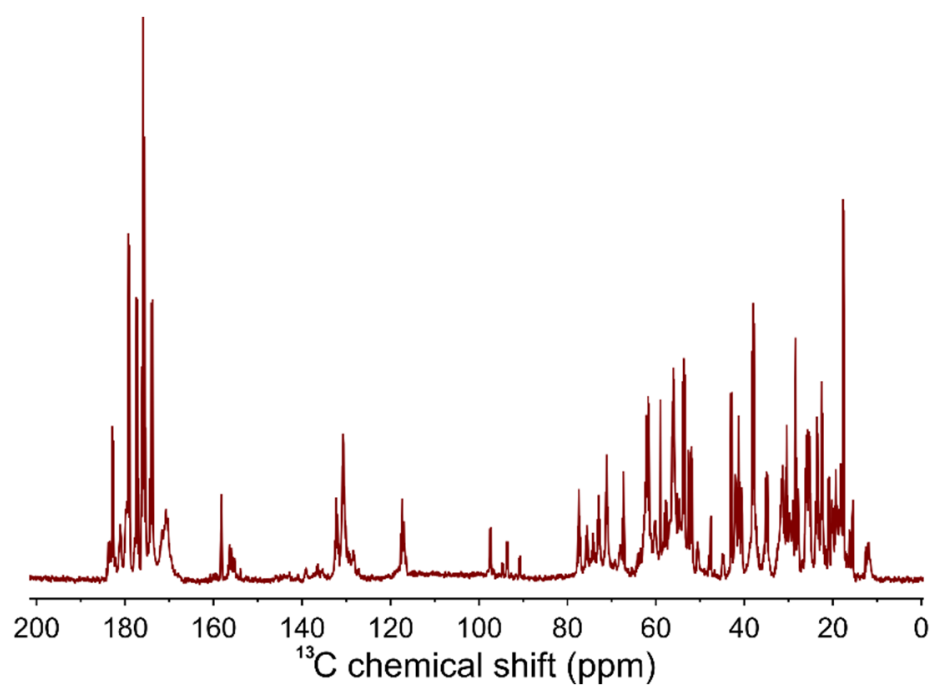

Figure S14. $176 \mathrm{MHz}{ }^{13} \mathrm{C}$ NMR spectrum of the ISOGRO supernatant in $\mathrm{D}_{2} \mathrm{O}(\mathrm{pH} 6.5)$. 


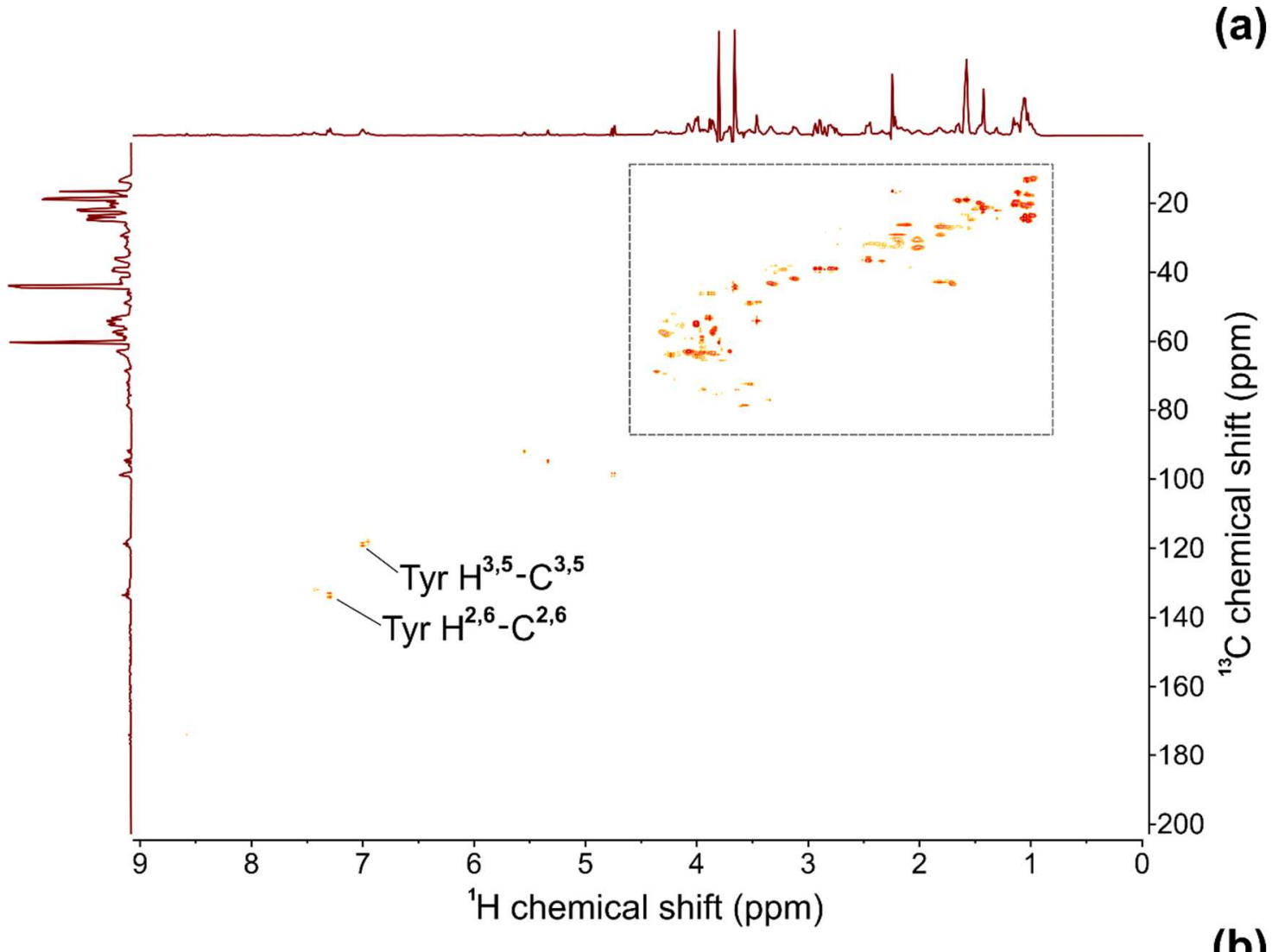

(a)

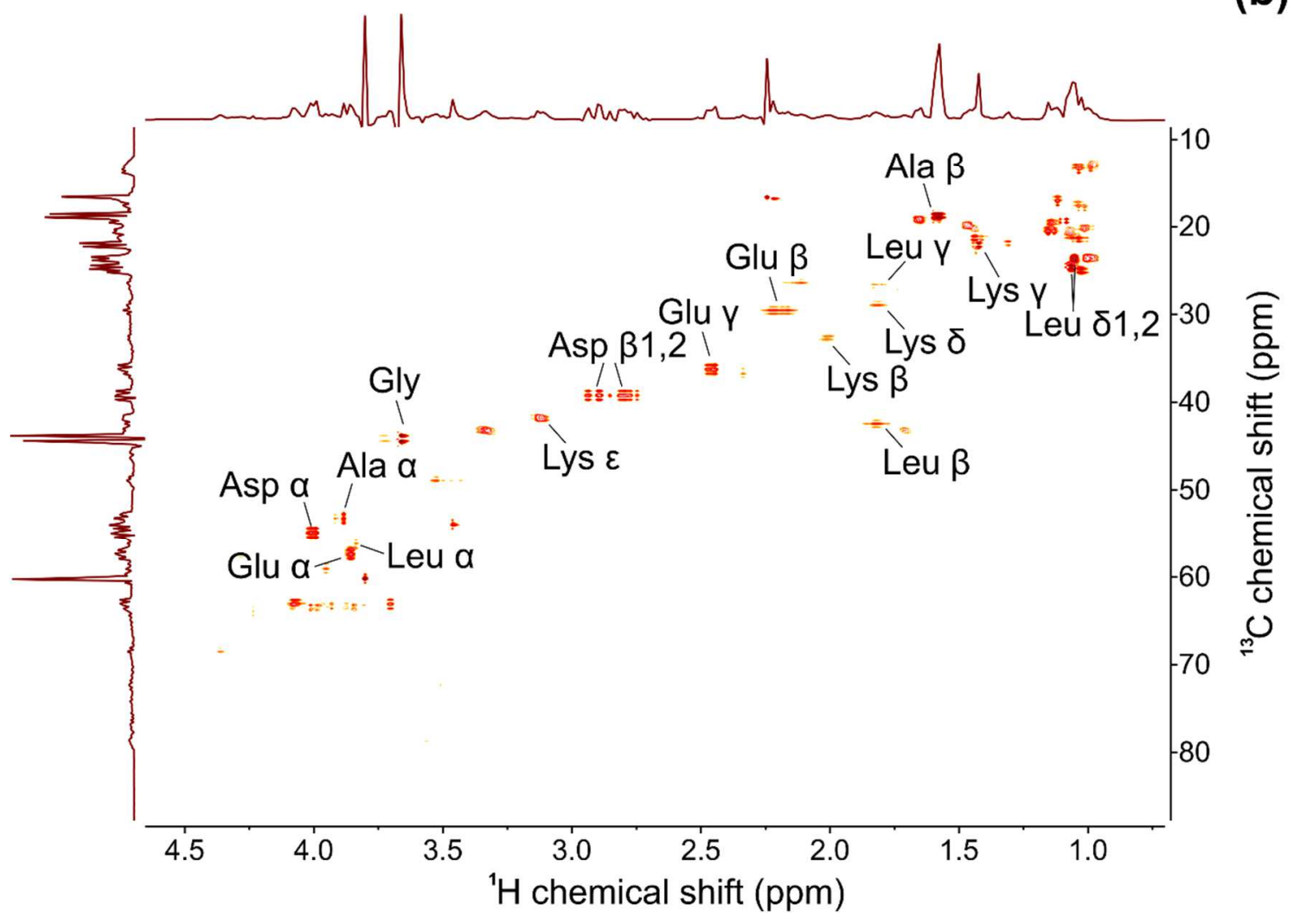

Figure S15. 400.13 MHz HSQC spectrum of ISOGRO supernatant in $\mathrm{D}_{2} \mathrm{O}$ (pH 6.5). The indirect dimension is sampled with 2048 transients over the 200 ppm spectral width, leading to approximately $20 \mathrm{~Hz}$ per point spectral resolution. Duration of the mixing period is $7 \mathrm{~ms}$. The acquisition time was ca. 42 hours. (a) Full spectrum. Correlations of the aromatic carbon and proton nuclei of tyrosine is marked. In (b) the region highlighted with a dashed square on subplot (a) is shown. 


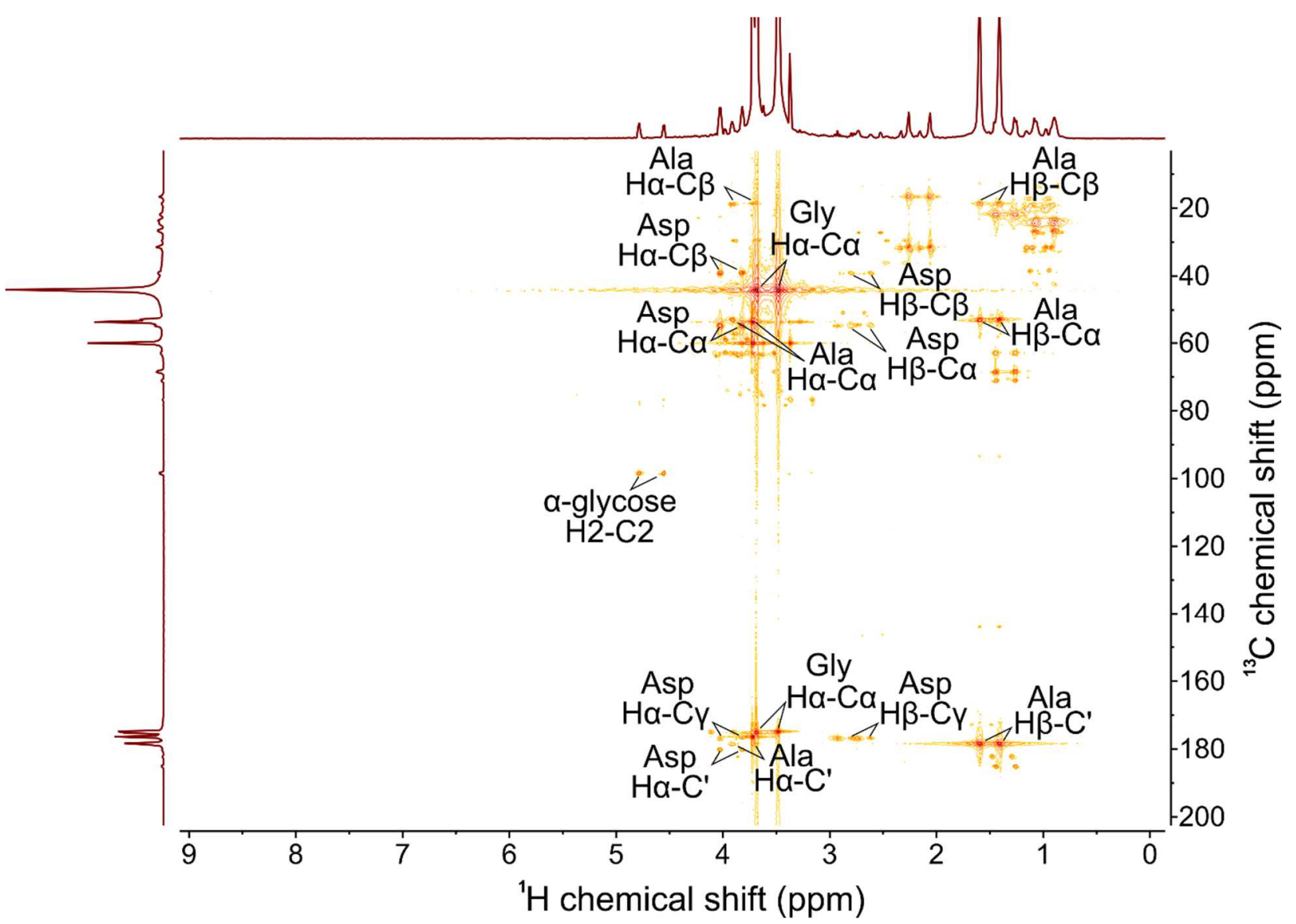

Figure $\mathrm{S} 16.700 \mathrm{MHz}{ }^{1} \mathrm{H}^{13}{ }^{13} \mathrm{CHMBC}$ spectrum of ISOGRO supernatant in $\mathrm{D}_{2} \mathrm{O}(\mathrm{pH}$ 6.5). To record the spectrum, Bruker's build-in pulse program "hmbcgplpndqf" was used, with J-filter from $3 \mathrm{~Hz}$ to $135 \mathrm{~Hz}$. The indirect dimension was sampled with 2048 transients over the 200 ppm spectral window, leading to approximately $34 \mathrm{~Hz}$ per point spectral resolution. Duration of the mixing period is $171 \mathrm{~ms}$. The acquisition time was ca. 18 hours. The acquired signals were multiplied by ${ }^{\circ} 90^{\circ}$ shifted squared sinusoidal window function in both dimensions. The spectrum contains presented by $1024 \times 512$ data points. 